\title{
Evolutionary Robotics and Neuroscience
}

\author{
Phil Husbands, Renan Moioli, Yoonsik Shim, Andy Philippides, \\ Patricia Vargas and Michael O'Shea
}

\section{Introduction}

When research in evolutionary robotics (ER) initially took off in the early 1990s, concerns over the brittleness of traditional AI techniques had recently led to a resurgence of interest in artificial neural networks (ANNs). This fact, coupled with the obvious (loose) analogy between robot control systems and biological nervous systems, meant that most ER researchers naturally gravitated towards neurocontrol systems (Husbands and Harvey 1992, Beer and Gallagher 1992, Harvey et al. 1994, Parisi and Nolfi 1993, Floreano and Mondada 1994). To many of those researchers neural networks also intuitively seemed to be more evolvable than other possible control substrates such as rules or programs - nodes and connections could be gradually changed or added or deleted in a flexible open-ended way (Harvey et al. 1993, Cliff et al. 1993, Beer and Gallagher 1992). In addition, from the earliest days, it has been noted that dynamical recurrent varieties of neural networks, many strongly biologically influenced, allow subtle dynamics that can be readily exploited in the generation of robust adaptive behaviour (de Garis 1990, Beer and Gallagher 1992, Harvey et al. 1993). Hence, from the outset artificial neural networks have been the predominant control system used in ER. Therefore the field has always had at least a tentative link with neuroscience. However, strands of work in which the link is more explicit have existed since the inception of the field and have continued to develop. They are the focus of this chapter.

The two main classes of ER research in which there is a strong tie with neuroscience are: work involving explicitly biologically inspired neural network controllers, often making use of cutting edge neuroscience, and research in which ER is used to develop or explore neural models aimed at answering specific questions in neuroscience. The former class is concerned with biologically inspired technology while the latter uses computational and robotic tools in scientific research. In some cases the boundary between the two classes can become rather blurred: often within a single piece of research both kinds of motivation can be found.

This chapter attempts to sketch a general map of the kind of relationships that exist between ER and neuroscience, using specific examples to illustrate the wide range of interactions between the two areas. A number of case studies are used to explore such relationships in more depth.

The following section lays out the kinds of interactions between ER and neuroscience that will be considered in the rest of the chapter. Following detailed descriptions of a number of case studies, focusing mainly on on-going research, the chapter concludes with a discussion of open issues and the prospects for such work. 


\section{Relationships between evolutionary robotics and neuroscience}

The two main classes of relevant research mentioned above - the use of neurobiologically inspired neural networks in ER, and the use of ER modelling in neurobiological research are rather broad, with many variations on each theme. In the following sections the scope of each of these categories will be fleshed out.

With the acceleration in fundamental neuroscientific research that has taken place since about the turn of the century, it has become ever clearer that the central nervous system (CNS) is a far more sophisticated and exotic system than that portrayed in the oldfashioned connectionist, electrical network, view of neural processing that has dominated the worlds of artificial neural networks and neural modelling (Dayan and Abbott 2001). The emerging picture is one of many interacting adaptive processes operating over different temporal and spatial scales. These new understandings, particularly with reference to behaviour generation, provide rich sources of inspiration, at many different levels of abstraction, for the development of artificial nervous systems for robots. A number of examples of such research are described later.

Natural adaptive and intelligent behaviour is the result of complex interactions between nervous system, body and environment. Biological neural systems are embodied and embedded. Because of this there has been a growing interest in using robots, employing on-board neural circuitry, to model aspects of animal behaviour. Such a methodology, the argument goes, can give deeper insights into behaviour generating neural mechanisms than disembodied models (Webb 2001, Beer 2003, Seth et al. 2004), as well as fresh perspectives on what it means to be a cognitive agent (Wheeler 2005). Like any modelling enterprise, there are many issues surrounding how to make robotic models, with their inevitable implementation constraints, properly relevant to biological enquiry. For a discussion of such matters see (Webb 2001, 2009) and AB (2009).

Evolutionary robotics has an important role to play in this context as it allows the exploration of whole classes of mechanisms, the automatic creation of working models when there are insufficient details to fully specify a system in advance, and a reduction in 'designer bias' (Harvey et al. 2005) through the use of an automatic search process which does not specify in advance what a solution should look like. Hence it has been recognized as a useful tool in investigating biological hypotheses (Husbands et al. 1997, Harvey et al. 2005, Floreano et al. 2008).

\subsection{Biological Inspiration}

Biological brains are often, quite rightly, posited as the most complex systems studied by science. As their mysteries are slowly unravelled, they provide many potential foci of inspiration for developing neuro-influenced controllers for robots. Four of the main such sources of inspiration that have found their way into ER research are:

- Neural architectures

- Intrinsic neural properties 
- Signalling modes

- Brain-body-environment dynamics

Each of these is briefly discussed in the remainder of this section. More detailed case studies involving one or more of these elements are covered later in the chapter.

Observations of the ways in which neural circuitry is organised in nature, particularly in relation to motor behaviours in invertebrates, have led to a number of powerful general architectures being adopted in some areas of ER, for instance work on legged locomotion. Such architectures impose constraints on the properties of neurons and the ways in which they can be connected, thus shaping and restricting the evolutionary search space. A pioneering example is the work of Beer and colleagues (Beer et al. 1989, Beer et al. 1997), who introduced an architecture for locomotion based on cross coupled subnetworks, inspired by the cockroach nervous system (Figure 1). Each leg is controlled by an identical (or near-identical) fully-connected subnetwork containing a small number of neurons (typically 5 or 6). These networks are connected to each other by both cross-body and same-side (intersegmental) wiring as illustrated in Figure 1. Variations on this insect-inspired architecture have proved very successful in developing locomotion controllers in many types of legged robots, including bipeds, quadrupeds, hexapods and octopods (Jakobi 1998, Kodjabachian and Meyer 1998, Vaughan et al. 2004, Reil and Husbands 2002).
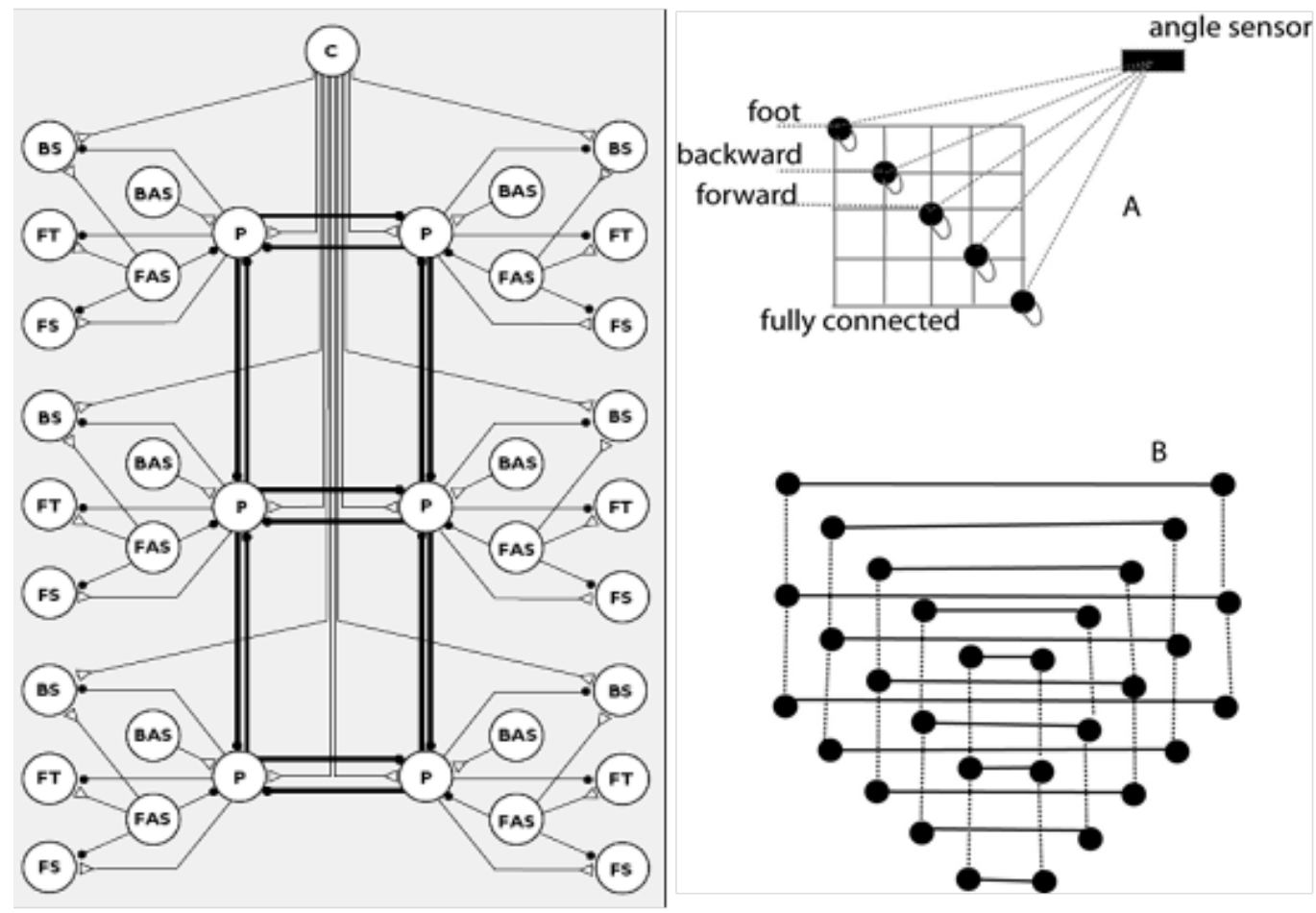

Figure1: LEFT: schematic diagram of a distributed neural network for the control of locomotion in a hexapod as used by Beer et al. (1989). Excitatory connections are denoted by open triangles and inhibitory connections are denoted by filled circles. C, command neuron; P, pacemaker neuron; FT, foot motor neuron; FS and BS, forward swing and backward swing motor neurons; FAS and BAS, forward and backward angle sensors. Reproduced with permission. RIGHT: generalised architecture using a fully 
connected dynamical network controller for each leg (A), cross coupled as shown (B). Solid lines are cross body connections, dashed lines are intersegmental connections.

Real neurons are highly sophisticated information processing devices, generally orders of magnitudes more complex than the crude caricatures employed in artificial neural networks. Their very sophistication means that detailed modelling becomes computationally very expensive, so it is inevitable that abstractions and simplifications should be made in any model or artificial analogue. However, by introducing elements of realistic neural properties, including dynamics, much can be gained. For instance, the particular brand of continuous-time recurrent neural networks (CTRNNs) used to great effect by many ER practitioners (Beer and Gallagher 1992, Beer 2003) are based on simple integrate and fire model neurons (Abbott and Kepler 1990) which were originally developed to approximate aspects of neural membrane potential dynamics without the expense of more detailed Hodgkin and Huxley (1952) style models. In contrast to the uniform nature of most ANNs, individual real neurons and synapses often have their own highly unique properties. In many identified motor circuits, particularly in invertebrates, nerve cells and synapses have widely varying intrinsic properties and behaviours (North and Greenspan 2007). Incorporating such heterogeneity into networks is particularly suited to the ER methodology and has often been successfully employed (Nolfi and Floreano 2000, Floreano et al. 2008). Many other intrinsic properties, such as spiking and oscillatory dynamics (Izhikevich 2007), homeostatic mechanisms (Davis 2006) and numerous forms of plasticity (e.g. Pinaud et al. 2006, Katz 1999), provide a rich vein of inspiration.

Until recently nearly all work in ANNs and computational neuroscience concentrated on the transmission of electrical signals between neurons via axons and dendrites. However, nervous systems are complex electrochemical systems with many nonelectrical signalling modes also in play. This fuller picture of neural information processing has inspired work in ER that makes use of analogues of chemical transmission. In particular, the notion of volume signalling, whereby neurotransmitters freely diffuse into a relatively large volume around a nerve cell, potentially affecting many other neurons irrespective of whether or not they are electrically connected (Gally et al. 1990, Wood and Garthwaite 1994), has been explored in a body of ER work (Husbands et al. 1998, Philippides et al. 2005b, Husbands et al. 2010). This exotic form of neural signalling, which involves modulation of neural and/or synaptic properties by the diffusing neurotransmitter, does not sit easily with classical connectionist (point-topoint) pictures of brain mechanisms and is forcing a radical re-think of existing theory (Dawson and Snyder, 1994; Philippides et al., 2000; Philippides et al., 2005a; Bullock et al., 2005; Katz, 1999). Other examples include the use of analogs of neuro-endocrine interactions in ER control architectures (Vargas et al. 2005), neural plasticity modulated by dopamine inspired mechanisms (Doya 2002) and the introduction of neuron-level homeostatic mechanisms in spiking plastic networks (DiPaolo 2003).

One of the driving forces behind the development of ER and other forms of 'New AI' has been the realisation that a proper understanding of intelligence must recognise the central role of embodiment (Varela et al. 1991, Brooks 1991, Clark 1999). The body is not just a passive vessel to be controlled. Rather, adaptive behaviour emerges out of a subtle interplay between brain, body and environment (Pfeifer and Bongard 2007). For instance, studying neural circuitry underlying the generation of rhythmic motor behaviour in isolation ignores the considerable advantage that can be obtained from 
incorporating the physical body and its environment - an approach that can significantly reduce the amount of information needed to develop successful motor patterns. ER has proved to be very useful in exploring and exploiting the interplay between neural, bodily and environmental dynamics in the development of efficient and robust behaviours

(Floreano et al. 2008).

Section 3 describes in some detail three examples of current work from the Centre for Computational Neuroscience and Robotics at Sussex University which mainly fall under the biological inspiration category. We say mainly, because although the studies are of biologically inspired neuro-robotic systems, there are elements of biological inquiry in each case.

\subsection{Biological Modelling}

Many types of model are now commonly used in science, including: the classical modelling of a target system at a particular level of abstraction according to some set of assumptions, and models as existence proofs - used to help to refute, or at least cast doubt on, certain claims about necessary conditions for phenomenon as well as demonstrating new possibilities. Models can also act as substitutes for theories where none exist, a situation common in cognitive science and biology, and, in the case of computational models, as a kind of animated thought experiment aimed at clarifying conceptual issues. ER has been used to develop examples of all of these types of models in relation to various issues in neuroscience; several illustrative cases are discussed later.

A slightly more abstract characterisation of the main classes of neurobiological modelling that have employed ER methods, which will be used in the remainder of the paper, uses the following three categories:

- Model tuning

- Model synthesis

- Development of Probing models

Model tuning here refers to adjusting or setting parameter values in an otherwise welldefined model (parameter fitting). There is a growing history of using search methods, including evolutionary techniques, to either fine tune values or set unknown values in scientific models, including neuroscientific models (e.g. Gerken et al. 2005, Gurkiewicz and Korngreen 2007). When the model is of a whole embodied behaviour generating system, evolutionary robotics can be an ideal tool to use in this context. In that case parameters might describe neural properties or aspects of sensors or bodies, or all of these things.

Model tuning is used when sufficient details of the target system are known to be able to define a parametric model at the desired level of abstraction. In cases where insufficient details are available (e.g. the connectivity of neural circuitry is unclear, or the number of neurons involved is unknown) model synthesis techniques can be employed. ER can be used for this purpose by searching a space of possible models, constrained by available knowledge, to find one that fits the data (e.g. generates appropriate behaviour). By attempting to fill in the blanks, a model synthesized in this way presents a set of hypotheses about the target system. For instance, details of how neural architectures and mechanisms underly behaviour generation. The hypotheses thus generated can then be 
subjected to empirical scrutiny in the original biological system. When knowledge of the target system or phenomenon is sparse, such synthesized models generally stand as existence proofs that serve to catalyse further debate and sharpen theories.

A kind of model that is closely related to the existence proof and that does not require any direct representational function, but is used in all branches of science, is the toy, or probing, model (Frigg \& Hartmann, 2008). Evolutionary robotics has increasingly been used to develop such models, which operate at a more abstract level than the other types listed above. They are not intended to represent a specific concrete target system or phenomenon, but to be used as simple vehicles for testing new tools and methods, preparatory to more detailed empirically based modelling (Hartmann, 1995). This is exactly the motivation Randy Beer gives for the strand of research which employs ER techniques to synthesize abstract models of agents engaged in 'minimally cognitive' behaviours (Beer, 2003). He describes his work on simple autonomous agents involved in categorizing objects within a behavioural context thus: "The intention here is not to propose a serious model of categorical perception, but rather to use this model agent to explore the implications of dynamical explanation for cognitive agents.” (Beer, 2003, p. 210), and exhorts us to "Think of this exercise, then, as a form of mental calisthenics, an intellectual warm-up for the dynamical analyses of a wider range of agents and behaviors." (Beer, 2003, p.210). An interesting associate of the toy model is the false model - a model of something known to be wrong - which can have a useful heuristic role in refining and developing 'true' models by elaborating their underlying assumptions (Wimsatt 2002).

For an area as difficult and underdeveloped as dynamical analyses and explanations of embodied situated agent behavior, which could have a significant affect on thinking in neuroscience, Beer's justification seems appropriate and pragmatic. The reason toy models are used in physics is the same reason Beer uses them: their relative tractability. ER can be a very powerful tool for building them.

A good example of using ER techniques for model tuning is the work of Ijspeert and colleagues (Ijspeert et al. 1999, Hallam and Ijspeert 2003) on models of the central pattern generator neural circuitry underlying swimming behaviours in the Lamprey. The starting point was a model at an intermediate level of abstraction devised by Ekeberg (Ekeberg et al. 1993,1995) which was based on neurophysiological data (Grillner et al. 1991). This model used networks of simple leaky integrator nodes to control a simulated Lamprey body. The nodes in the networks can be thought of as populations of neurons and the connections between them as general pathways in the Lamprey's spinal cord. This model had a well-defined set of parameters that described individual node and connection properties. Ekeberg hand-designed a set of specific parameter values that were able to reproduce some of the real Lamprey's behaviours to a good level of accuracy (Ekeberg et al. 1995). Ijspeert and colleagues used a genetic algorithm to search the parameter space of this model and were able to find several combinations different from Ekeberg's handdesigned set that reproduced the biological data better. Since the ER generated solutions were all essentially variations of Ekeberg's model, the work also showed that the original model was fairly robust to differences in parameter values (Hallam and Ijspeert 2003). Ijspeert also used ER as a model synthesis technique by relaxing the constraints imposed by Ekeberg's parametric model and searching the resulting space of possible models. They found a number of alternative models that were also able to reproduce the biological data. 
A more recent example of using ER for model synthesis is the work of Izquierdo and Lockery (2010). They used ER methods to develop a model of the neural mechanisms underlying klinotaxis, a common form of chemotaxis, in C. Elegans. Previous neural models of chemotactic behaviour in nematodes focused on the other strategy they commonly use: klinokinesis, in which the direction of movement is governed by a biased random walk (Ferree and Lockery, 1999). Klinotaxis involves movements in which the direction of locomotion in a chemical gradient closely follows the line of steepest ascent. The differences between the two forms of behaviour imply a distinctive neural network controlling klinotaxis. This network has not yet been identified and no hypothetical model existed before Izquierdo and Lockery's work. They used an evolutionary algorithm to generate neural networks that exhibited klinotaxis in a simple idealised physical model of C. Elegans. Sensory inputs and motor outputs of the model networks were constrained to match empirical data as were other aspects of a hypothesized network architecture. Motor neurons were modelled as simple leaky integrators as used in CTRNNs (Beer 1995). The parameters of the resulting network were evolved to discover working instances of the network that could then stand as hypotheses about the mechanisms at play in C.Elegans. They discovered that a minimalistic neural network, comprised of an ONOFF pair of chemosensory neurons and a pair of neck muscle motor neurons, is sufficient to generate realistic klinotaxis behaviour. Importantly, emergent properties of model networks reproduced other experimental observations that they were not designed to fit, suggesting that the model may be operating according to principles similar to those of the biological network. A large number of successful networks were analysed and this revealed a novel neural mechanism to allow asymmetric turning behaviour (a kind of mutual inhibition between motor neurons is achieved simply by shifting the sigmoidal input-output function of the motor neurons relative to the dynamic range of the oscillatory input driving the nematode's head sweeps). The authors stress that this mechanism provides a testable hypothesis that is likely to accelerate the discovery and analysis of the biological circuitry for chemotaxis in C. Elegans.

An interesting example of ER used in a probing model context is given by Seth (2005). The aim of the work was to develop methods based on Granger causality (Granger 1969) to enable analysis of causal interactions occurring within behaviour generating neural mechanisms. This requires detailed simultaneous temporal data from several sites in some relevant neural circuitry, in practice not at all easy to gather from intact biological neural system. Rather than try and develop the method (termed 'causal connectivity analysis') using data somehow collected from a biological context, Seth very sensibly opted to experiment with the ideas in an abstract simulation. This is a classic case of the kind of situation in which probing models can be very useful, as discussed earlier. He used a genetic algorithm to develop model neural networks optimized for controlling target fixation in a simulated head-eye system, in which the structure of the environment could be experimentally varied. Causal connectivity analysis of a number of networks evolved in various contexts within this framework gave novel insights into neural mechanisms underlying sensorimotor coordination. He demonstrated that networks underlying relatively rich adaptive behaviour showed a higher density of causal interactions, as well as a stronger causal flow from sensory inputs to motor outputs, than networks generating relatively simple behaviours. In addition he showed that this style of analysis can predict the functional consequences of network lesions. The methods developed using this probing model were powerful enough to suggest that causal connectivity analysis, and similar techniques, could have useful applications in the 
analysis of real neural dynamics, a line of enquiry that has been followed by Seth and colleagues with some success (e.g. Seth et al. 2011, Barnett and Seth 2011).

Suzuki et al.'s (2005) work on whether or not proprioceptive motor information resulting from the generation of actions is necessary for the development of normal, visually-guided behavior is another example of ER being used to explore an explicitly neuroscientific question. In an experiment inspired by Held and Hein's (1963) work on cats, two initially identical evolved robots were compared. One was left free to move in a square environment while the other was forced to move along trajectories imposed externally, but was free to control its camera position. The visual receptive fields and behaviours of the passive robot significantly differed from those of the active robot. Further analysis revealed that passive robots became over-sensitivity to features that were not functional to their normal behaviour and which interfered with other dominant features in the visual field. This lead to a hypothesis that some pathological behaviours seen in animals might have roots in similar developmental deficiencies.

\section{Neuroscience-inspired ER Case Studies}

This section illustrates in more detail how ideas from empirical and theoretical neuroscience can provide powerful inspiration for work in ER by focusing on three examples of current work from our lab at Sussex University.

\subsection{Volume Signalling: GasNets}

A good example of ER research drawing strongly on inspiration from neuroscience is that involving the class of artificial neural networks developed to explore an analogue of volume signalling - so-called GasNets (Husbands et al., 1998). These take particular inspiration from nitric oxide (NO) signalling (Gally et al., 1990). They comprise a fairly standard artificial neural network augmented by a chemical signalling system based on a diffusing virtual gas which can modulate the response of other neurons. Because there was (and still is) insufficient knowledge of the biological systems to completely define artificial systems working on similar principles, the networks were developed to be used within an evolutionary robotics context. Thus a number of GasNet variants, inspired by different aspects of real nervous systems, have been explored as artificial nervous systems for mobile autonomous robots. They are being investigated as potentially useful engineering tools, including as modules in complex robot control systems (Vargas et al., 2009), while a related strand of more detailed modelling work is aimed at gaining helpful insights into biological systems (Philippides et al., 2000, 2003, 2005a).

By analogy with biological neuronal networks, GasNets incorporate two distinct signalling mechanisms, one 'electrical' and one 'chemical'. The underlying 'electrical' network is a discrete time step, recurrent neural network with a variable number of nodes. These nodes are connected by either excitatory or inhibitory links with the output, $O_{i}^{t}$, of node $i$ at time step $t$ determined by the following equation.

$$
O_{i}^{t}=\tanh \left[k_{i}^{t}\left(\sum_{j \in \Gamma_{i}} w_{j i} O_{j}^{t-1}+I_{i}^{t}\right)+b_{i}\right]
$$


where $\Gamma_{i}$ is the set of nodes with connections to node $i$ and $w_{j i}= \pm 1$ is a connection weight. $I_{i}^{t}$ is the external (sensory) input to node $i$ at time $t$, and $b_{i}$ is a genetically set bias. Each node has a genetically set default transfer function gain parameter, $k_{i}^{0}$, which can be altered at each time-step according to the concentration of diffusing 'gas' at node $i$ to give $k_{i}^{t}$ (as described later).

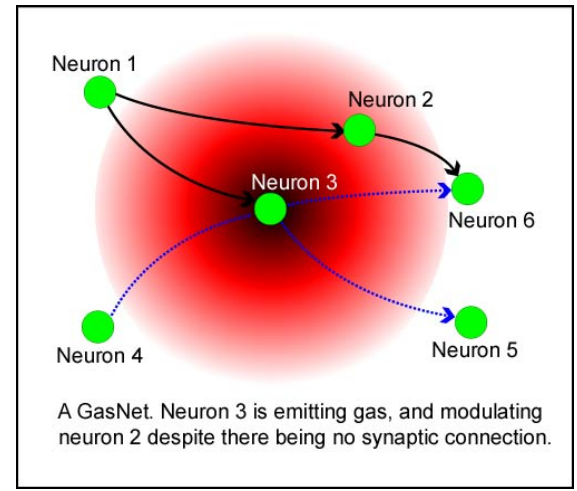

Figure 2: A basic GasNet showing excitatory (solid) and inhibitory (dashed) 'electrical' connections and a diffusing virtual gas creating a 'chemical' gradient.

In addition to this underlying network in which positive and negative 'signals' flow between units, an abstract process loosely analogous to the diffusion of gaseous modulators is at play. Some units can emit virtual 'gases' which diffuse and are capable of modulating the behaviour of other units by changing their transfer functions. The networks occupy a 2D space; the diffusion processes mean that the relative positioning of nodes is crucial to the functioning of the network. Spatially, the gas concentration varies as an inverse exponential of the distance from the emitting node with spread governed by a parameter, $r$, genetically set for each node, which governs the radius of influence of the virtual gas from the node as described by the equations below and illustrated in Figure 2. The maximum concentration at the emitting node is 1.0 and the concentration builds up and decay linearly as dictated by the time course function, $T(t)$, defined below.

$$
\begin{gathered}
C(d, t)=\left\{\begin{array}{cc}
e^{-2 d / r} \times T(t) & d<r \\
0 & \text { else }
\end{array}\right. \\
T(t)=\left\{\begin{array}{cc}
t=0 \\
\min \left(1,\left(T(t-1)+\frac{1}{s}\right)\right) & \text { emitting } \\
\max \left(0,\left(T(t-1)-\frac{1}{s}\right)\right) & \text { not emitting }
\end{array}\right.
\end{gathered}
$$


where $C(d, t)$ is the concentration at a distance $d$ from the emitting node at time $t$ and $s$ (controlling the slope of the function $T$ ) is genetically determined for each node. The range of $s$ is such that the gas diffusion timescale can vary from 0.5 to 0.09 of the timescale of 'electrical' transmission (i.e. a little slower to much slower). The total concentration at a node is then determined by summing the contributions from all other emitting nodes (nodes are not affected by their own emitted gases to avoid runaway positive feedback). The diffusion process is modelled in this simple way to provide extreme computational efficiency, allowing arbitrarily large networks to be run very fast - a very useful property in the context of evolutionary search.

For mathematical convenience, in the original basic GasNet there are two 'gases', one whose modulatory effect is to increase the transfer function gain parameter $\left(k_{i}^{t}\right)$ and one whose effect is to decrease it. It is genetically determined whether or not any given node will emit one of these two gases (gas 1 and gas 2), and under what circumstances emission will occur (either when the 'electrical' activation of the node exceeds a threshold, or the concentration of a genetically determined gas in the vicinity of the node exceeds a threshold; note these emission processes provide a coupling between the electrical and chemical mechanisms). The concentration-dependent modulation is described by the following equation, with transfer function parameters updated on every time step as the network runs:

$$
k_{i}^{t}=k_{i}^{0}+\alpha C_{1}^{t}-\beta C_{2}^{t}
$$

where $k_{i}^{0}$ is the genetically set default value for $k_{i}, C_{1}^{t}$ and $C_{2}^{t}$ are the concentrations of gas 1 and gas 2 respectively at node $i$ on time step $t$, and $\alpha$ and $\beta$ are constants such that $k_{i}^{t} \in[-4,4]$. Thus the gas does not alter the electrical activity in the network directly but rather acts by continuously changing the mapping between input and output for individual nodes, either directly or by stimulating the production of further virtual gas. The general form of diffusion is based on the properties of a (real) single source neuron as modelled in detail in Philippides et al. (2000; 2003). The modulation chosen is motivated by what is known of NO modulatory effects at synapses (Baranano et al., 2001). For further details see (Husbands et al., 1998; Philippides et al., 2005, Husbands et al. 2010).

When they were first introduced, GasNets were demonstrated to be significantly more evolvable than a variety of standard ANNs on some noisy visually guided evolutionary robotics tasks (Husbands, 1998; Husbands et al., 1998). Typically the increase in evolvability, in terms of number of fitness evaluations to a reliable good solution, was an order of magnitude or more. The solutions found were often very lean with few nodes and connections, typically far fewer than were needed for other forms of ANN (Husbands et al., 1998). But the action of the modulatory gases imbued such networks with intricate dynamics: they could not be described as simple. Oscillatory sub-networks based on interacting 'electrical' and 'gas' feedback mechanisms acting on different timescales were found to be very easy to evolve and cropped up in many forms, from CPG circuits for locomotion (McHale and Husbands, 2004) to noise filters and timing mechanisms for visual processing (Husbands et al., 1998, Smith et al., 2002). GasNets appeared to be particularly suited to noisy sensorimotor behaviours which could not be solved by simple reactive feedforward systems, and to rhythmical behaviours. 
Two recent extensions of the basic GasNet, the receptor and the plexus models, incorporated further influence from neuroscience (Philippides et al. 2005b). In the receptor model modulation of a node is now a function of gas concentration and the quantity and type of receptors (if any) at the node. This allows a range of site specific modulations within the same network. In the plexus model, inspired by a type of NO signalling seen in the mammalian cerebral cortex (Philippides et al. 2005a), the emitted gas 'cloud', which now has a flat concentration, is no longer centred on the node controlling it but at a distance from it. Both these extended forms proved to be significantly more evolvable again than the basic GasNet. Other varieties include nonspatial GasNets where the diffusion process is replaced by explicit gas connections with complex dynamics (Vargas et al. 2009) and version with other forms of modulation and diffusion (Husbands et al. 2010). In order to gain insight into the enhanced evolvability of GasNets, detailed comparative studies of these variants with each other, and with other forms of ANN, were performed using the robot task illustrated in Figure 3 (Philippides et al. 2005b,Husbands et al. 2010).

The question naturally arises as to why the GasNet and variants are more evolvable. Intriguingly, in a comprehensive study Smith et al. (2003), found no explanation for increased GasNet evolvability in terms of fitness landscape properties (neutrality, epistasis etc) apart from at high fitness values. There it was argued that the key to understanding the improvement of the GasNet was to analyse its behaviour at a higher level of abstraction. In particular it was shown that the temporal dynamics of the GasNet seemed to make it relatively easy to tune the networks to the time-scales needed in the task (Smith et al. 2002). Similar high level analyses of the spatial structure of successful GasNets and variants, led to the hypothesis that it was the level of coupling between the electrical and gas signalling systems that was key. In particular that successful evolution came through the systems being flexibly coupled: neither independent of each other nor too tightly bound, allowing one system to be 'tuned' against the other without causing catastrophic destructive interference (Philippides et al. 2005b). Throughout, however, it was clear that these factors did not act in isolation and that it is the modulatory effect of the gas that lends the networks their adaptivity. This leads to three linked hypotheses on why the GasNets evolve faster:

- The action of gas over multiple different timescales from the electrical activity introduces rich dynamics which can be exploited

- The spatial embedding of the networks serves to (flexibly) couple two interacting signalling systems

- The particular modulatory effects are key to evolvability

These hypotheses were examined in an extended empirical studies, focusing on a comparison of variants of the basic GasNet formed by imposing various constraints on spatial, temporal and modulatory properties.

\section{Comparative Study}

Nine GasNet variants were compared in order to probe the questions outlined above. These implement a range of constraints affecting spatial, temporal and modulatory factors, and are described in Table 1. 


\begin{tabular}{|c|c|c|}
\hline \# & name & Description \\
\hline 1 & gnet & Basic gasnet as described in previous section. \\
\hline 2 & nchem & Basic gasnet but with all chemicals inactive. \\
\hline 3 & gnetN & Basic gasnet with no diffusion dynamics, i.e. $T(t)=1$, for all $t$ (see Eqn. 3 ). \\
\hline 4 & gnetNw & $\begin{array}{l}\text { The same as gnetN but with } T(t)=w \text { where } w \in\{0,1,2\} \text { is a 'gas weight' } \\
\text { genetically set for each node. }\end{array}$ \\
\hline 5 & flatR & $\begin{array}{l}\text { The same as gnet except the gas concentration within the genetically } \\
\text { set radius for each emitter is flat with no gradient (the term } e^{-2 d / r} \text { in Eq. } 2 \\
\text { is replaced by } e^{-1} \text { ). }\end{array}$ \\
\hline 6 & flatRN & $\begin{array}{l}\text { The same as flatR except without diffusion dynamics, i.e. } T(t)=1 \text {, for all } t \\
\text { (see Eqn. 3). }\end{array}$ \\
\hline 7 & flatE & $\begin{array}{l}\text { The same as flatR except the influence of the gas is not confined to the } \\
\text { genetically set radius of influence for a node but now extends } \\
\text { everywhere. }\end{array}$ \\
\hline 8 & flatEN & The same as flatE but without diffusion dynamics. \\
\hline 9 & AddMod & $\begin{array}{l}\text { The most radical variant where the multiplicative modulation of the basic } \\
\text { GasNet is replaced by an additive modulation as described by Eq. } 5 \text { (i.e., } \\
\text { the gas no longer modulates the transfer function gain parameter but } \\
\text { instead modulates an additional additive bias term). }\end{array}$ \\
\hline
\end{tabular}

Table 1: The GasNet variants used in a comparative study.

$$
O_{i}^{t}=\left[k_{i}^{0}\left(\sum_{j \in \Gamma_{i}} w_{j i} O_{j}^{t-1}+I_{i}^{t}\right)+b_{i}+\gamma_{i}\left(C_{1}^{t}-C_{2}^{t}\right)\right]
$$

The task used in the studies is illustrated in Figure 3. Starting from an arbitrary position and orientation in a black-walled arena, a robot equipped with a forward facing camera must navigate under extremely variable lighting conditions to one shape (a white triangle) while ignoring the second shape (a white rectangle). The robot must successfully complete the task over a series of trials in which the relative position and size of the shapes varies. Both the robot control network and the robot sensor input morphology, i.e. the number and positions of the camera pixels used as input and how they were connected into the network, were under evolutionary control as shown in Figure 3. The network architecture (including number of nodes) and all properties of the nodes and connections and gas diffusion parameters were set by an evolutionary search algorithm. Because of the noise and variation, and limited sensory capabilities (only very few pixels are used), this task is challenging, requiring robust, general solutions. The gantry robot shown in the figure was used. Evolution took place in a special validated simulation of the robot and its environment. 

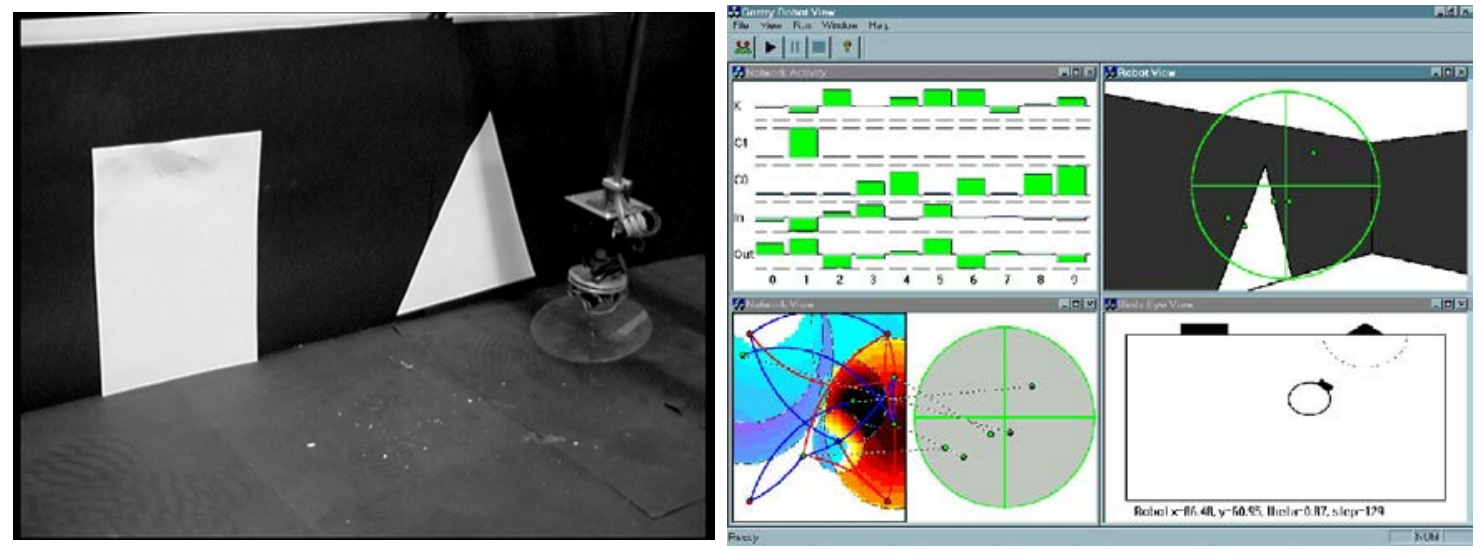

Figure 3: LEFT: The gantry robot. A CCD camera head moves at the end of a gantry arm allowing full 3D movement. In the study referred to in the text 2D movement was used, equivalent to a wheeled robot with a fixed forward pointing camera. A validated simulation was used: controllers developed in the simulation work at least as well on the real robot. RIGHT: The simulated arena and robot. The bottom right view shows the robot position in the arena with the triangle and rectangle. Fitness is evaluated on how close the robot approaches the triangle. The top right view shows what the robot 'sees', along with the pixel positions selected by evolution for visual input. The bottom left view shows how the genetically set pixels are connected into the control network whose gas levels are illustrated. The top left view shows current activity of nodes in the GasNet.

In all cases networks were encoded on a variable sized genotype coding for a variable number of nodes. A genotype consisted of an array of integer variables, each lying in the range $[0,100]$. For continuous variables, the phenotype value is obtained by normalising the genotype value to lie in the range $[0.0,1.0]$ and multiplying by the relevant variable range. For nominal values, such as whether or not the node has a visual input, the phenotype value $=$ genotype value $\mathrm{MOD} \mathrm{N}_{\text {nom }}$, where $\mathrm{N}_{\text {nom }}$ is the number of possible nominal values, and MOD is the binary modular division operator. Each node in the network had between 19 and 21 variables associated with it, depending on which network variant it described. These define the node's position on a 2D plane; how the node connects to other nodes on the plane with either excitatory (weight +1 ) or inhibitory (weight -1) connections; whether or not the node has visual input, and if it does the coordinates of the camera pixel it takes input from, along with a threshold below which input is ignored; whether or not the node has a recurrent connection; whether and under what circumstances the node can emit a gas and if so which gas it emits; and a series of variables describing the gas emission dynamics (maximum range, rate of emission and decay etc). All variables were under evolutionary control. Four of the nodes are assigned as motor nodes (forward and backward nodes for the left and right motor, with motor speeds proportional to the output of the relevant forward node minus the output of the relevant backward node). See Husbands et al. $(1998,2010)$ for full details.

16 evaluations were carried out on an individual network, with scores $\mathrm{f}_{\mathrm{i}}$ calculated on the fraction of the initial robot-triangle distance that the robot moves towards the triangle by the end of the evaluation; a maximum score of 1.0 is obtained by getting within $10.0 \mathrm{~cm}$ of the triangle at any time during the evaluation. The controller only receives visual input; reliably getting to the triangle over a series of trials with different starting conditions, different relative positions of the triangle and rectangle, and under 
very noisy lighting, can only be achieved by visual identification of the triangle. The evaluated scores are ranked, and the fitness $\mathrm{F}$ is the weighted sum of the $\mathrm{N}=16$ scores, with weight proportional to the inverse ranking $\mathrm{i}$ (ranking is from 1 to $\mathrm{N}$, with $\mathrm{N}$ as the lowest score):

$$
F=\frac{\sum_{i=1}^{N} i f_{i}}{\sum_{i=1}^{N} i}=\frac{2}{N(N+1)} \sum_{i=1}^{N} i f_{i}
$$

Note the higher weighting on the poorer scores provides pressure to do well on all evaluations; a solution scoring 50\% on every evaluation has fitness nearly 4 times that of one scoring $100 \%$ on half of the evaluations and zero on the other half.

A geographically distributed asynchronous updating evolutionary algorithm was used (Collins and Jefferson 1991, Husbands 1992, Husbands et al. 1998), with a population size of 100 arranged on a 10 x10 grid. Parents were chosen through rank-based roulettewheel selection on the mating pool consisting of the 8 nearest neighbours to a randomly chosen grid-point. A mutated copy of the parent was placed back in the mating pool using inverse rank-based roulette-wheel selection. Three mutation operators were applied to solutions during evolution. Each integer in the genotype string had a $10 \%$ probability of mutation in a Gaussian distribution around its current value (for certain genes, $20 \%$ of its mutation will be random jumps within the full possible range). There was also an addition operator, with a $4 \%$ chance per genotype of adding one neuron to the network by inserting a block of random values describing each of the new node's properties, and a deletion operator, also with a 4\% chance per genotype of deleting one randomly chosen neuron from the network. An evolutionary run is terminated when a perfect score has been achieved in 10 successive generations, or after 10,000 generations if the former criteria is not met.

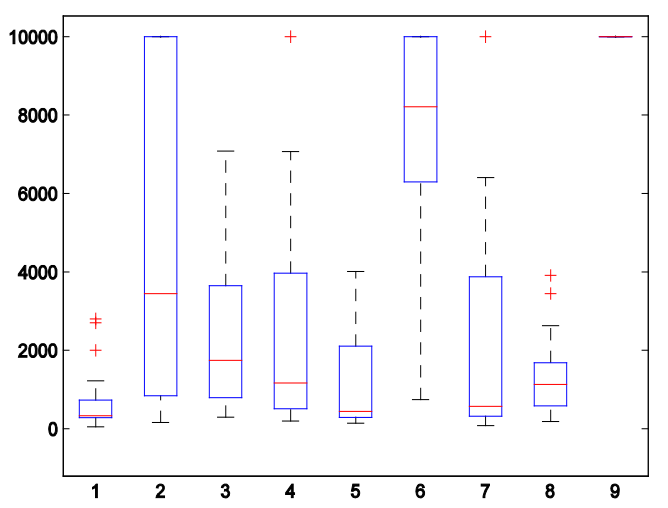

Figure 4: Boxplot summarising results of the comparative study. The $X$ axis refers to the network type numbers as shown in Table 1. The $Y$ axis shows generations to success as defined by the stopping criteria explained in the text. The horizontal line within each box is the median, the top and bottom of the box show the 75 th and $25^{\text {th }}$ percentiles respectively, the whiskers extend to extreme points of the data not considered outliers (as defined by Rosner's test), with outliers plotted individually. Forty runs of each network type are included. 


\begin{tabular}{|l|l|l|l|l|l|l|l|l|}
\hline $\begin{array}{l}\text { Sig } \\
\text { diff? }\end{array}$ & $\mathbf{1}$ & $\mathbf{2}$ & $\mathbf{3}$ & $\mathbf{4}$ & $\mathbf{5}$ & $\mathbf{6}$ & $\mathbf{7}$ & $\mathbf{8}$ \\
\hline $\mathbf{1}$ & $\mathrm{n}$ & $\mathrm{Y}$ & $\mathrm{Y}$ & $\mathrm{n}$ & $\mathrm{n}$ & $\mathrm{Y}$ & $\mathrm{n}$ & $\mathrm{n}$ \\
\hline $\mathbf{2}$ & $\mathrm{Y}$ & $\mathrm{n}$ & $\mathrm{n}$ & $\mathrm{n}$ & $\mathrm{n}$ & $\mathrm{n}$ & $\mathrm{n}$ & $\mathrm{n}$ \\
\hline $\mathbf{3}$ & $\mathrm{Y}$ & $\mathrm{n}$ & $\mathrm{n}$ & $\mathrm{n}$ & $\mathrm{n}$ & $\mathrm{n}$ & $\mathrm{n}$ & $\mathrm{n}$ \\
\hline $\mathbf{4}$ & $\mathrm{n}$ & $\mathrm{n}$ & $\mathrm{n}$ & $\mathrm{n}$ & $\mathrm{n}$ & $\mathrm{Y}$ & $\mathrm{n}$ & $\mathrm{n}$ \\
\hline $\mathbf{5}$ & $\mathrm{n}$ & $\mathrm{n}$ & $\mathrm{n}$ & $\mathrm{n}$ & $\mathrm{n}$ & $\mathrm{Y}$ & $\mathrm{n}$ & $\mathrm{n}$ \\
\hline $\mathbf{6}$ & $\mathrm{Y}$ & $\mathrm{n}$ & $\mathrm{n}$ & $\mathrm{Y}$ & $\mathrm{Y}$ & $\mathrm{n}$ & $\mathrm{Y}$ & $\mathrm{Y}$ \\
\hline $\mathbf{7}$ & $\mathrm{n}$ & $\mathrm{n}$ & $\mathrm{n}$ & $\mathrm{n}$ & $\mathrm{n}$ & $\mathrm{Y}$ & $\mathrm{n}$ & $\mathrm{n}$ \\
\hline $\mathbf{8}$ & $\mathrm{n}$ & $\mathrm{n}$ & $\mathrm{n}$ & $\mathrm{n}$ & $\mathrm{n}$ & $\mathrm{Y}$ & $\mathrm{n}$ & $\mathrm{n}$ \\
\hline
\end{tabular}

Table 2: Summary of tests for differences between evolvability (generations to consistent success). Distributions for network types 1-8 were tested against each other using pair-wise Wilcoxon RankSum tests adjusted for multiple comparisons using the Dunn-Sidak procedure. Cell entries state whether or not there is a significant difference between the two distributions in question $(\mathbf{p}<0.05)$.

Results of the comparative study are summarised in Figure 4. A quick glance suggests that the basic gasnet (group 1) is the most consistently evolvable with group 9 (AddMod) clearly the worst (no runs were successful). Group 2 (nchem), in which gas effects are turned off, performs poorly on most runs, although, like most other variants, some runs produce good solutions relatively quickly. Most other network types without diffusion dynamics, thereby robbed of rich temporal properties, including multiple timescales, perform relatively poorly (groups 3,4,6). However, the relatively good performance of group 8, without dynamics, especially compared to group 7, which has dynamics, suggests that the story is not quite as simple as it might at first appear. Since it is not possible to assume the data distributions are normal, non parametric statistical procedures were used to test for significant differences between the network types. A Kruskal-Wallis test performed on the whole data set (all 9 groups) revealed highly significant differences between the distributions $(\mathrm{p}<10-14)$. Pair-wise Wilcoxon RankSum tests, adjusted for multiple comparisons using the Dunn-Sidak procedure for controlling type-1 statistical error, were used to further probe the differences between the distributions. These tests showed that all network types, except group 6 (flatRND), were significantly more evolvable (in terms of generations to consistent success) than group 9 (AddMod). Since the Dunn-Sidak procedure is necessarily conservative and becomes more so as the number of groups increases, pairwise comparisons were recalculated for all network types except AddMod (i.e. groups 1-8). The results of these comparisons are shown in Table 2.

The results reveal the importance of the dynamics conferred by the diffusing gases. The basic GasNet (group 1) is significantly more evolvable than the variant with the gas turned off (group 2) as well as the variants with the gas operating but without dynamics (groups 3 and 4). It is also significantly more evolvable than the variant with the gas operating but with neither a concentration gradient nor dynamics (group 6). However, there is one group without gas dynamic that the basic GasNet is not significantly more evolvable than: group 8 (flatEN), of which more later. The version of the GasNet with diffusion dynamics but without a concentration gradient (group 5, flatR) performs fairly well with a low minimum and median, but the fairly high spread of results means that it is not as reliably evolvable as the basic GasNet. There is a similar story for group 7 (flatE) but its reliability is even worse; it should be noted that this restricted form of GasNet has similarities with various network models of neuromodulation that use global modulator signals (Doya 2002). 
Although these results suggest there is more to the GasNet's evolvability than the multiple timescales provided by the gas diffusion dynamics, they do add a certain amount of weight to previous suggestions (Philippides et al. 2005b) that their easily tuneable dynamics is an important part of their success (as well as to more general claims about the importance of dynamics in the generation of behaviour).

Even more obvious is the role of the type of modulation used - additive modulation proved to be useless (group 9). The multiplicative modulation employed in all other variants is able to assert a much more drastic influence on a node, being able to radically change the transfer function by altering the gain $\mathrm{k}_{\mathrm{i}}^{\mathrm{t}}$ (Equation 1 ) - for instance flipping the slope from positive to negative or making it flat. These kinds of radical changes were dynamically employed in most successful GasNets and were at the heart of mechanisms, such as oscillators, used to produce stable reliable behaviour in the face of significant noise (Husbands et al. 1998, Smith et al. 2002). Additive modulation, which acts at the same level as a node input or bias, could not produce strong enough effects to generate stable behaviour. When GasNets were first introduced (Husbands 1998) an alternative node transfer function was successfully used along with an exponential modulation (changing exponents in a polynomial transfer function) that allowed potentially large alterations to the transfer function, which seems to be necessary for effective evolution. These kinds of (multiplicative or exponential) modulations may well confer evolutionary advantages by allowing network nodes to be sensitive to different ranges of input (internal and sensory) in different contexts. For instance, in one (behavioural) context an input node may need to be sensitive to a range of low sensor values while in another it is required to be sensitive to a range of high values. Changing a node's gain through multiplicative modulation allows its sensitivity to be adjusted in an appropriate way.

The spatial embedding of the networks also appears to play a role in producing the most effective coupling between the two distinct signalling processes ('electrical' and 'chemical'). By exploiting a loose, flexible coupling between the two processes, it is possible to significantly reduce destructive interference between them, allowing one to be 'tuned' against the other while searching for good solutions. It has been suggested that similar forces may be at play in spiking networks, where sub-threshold and spiking dynamics interact with each other, which have been evolved to drive vision-based robot behaviours (Floreano and Mattiussi 2001, Floreano et al., 2008). In the most successful varieties of GasNet, dynamics, modulation and spatial embedding act in concert to produce highly evolvable degenerate (Tononi et al. 1999) networks.

\subsection{Coupled Oscillator Networks and Minimal Cognition}

From shortly after the birth of modern neuroscience at the turn of the last century, researchers have looked at neuronal dynamics from an oscillatory perspective (Berger 1929). The consensus nowadays is that cognitive processes have a close non-trivial relationship to neuronal rhythms and oscillations (Buzsaki 2006). The importance of considering temporal relations among groups of neurons, modulated by external influences and/or sustained by internal mechanisms, has been stressed by various researchers in recent years (Engel et al. 2001, Singer 1999, Konig et al. 1996). According to Varela et al. (2001) it is essential to investigate the temporal dynamics of neural networks in order to 
understand the emergence and integration of neuronal assemblies by means of synchronisation. These dynamic assemblies, which are related to large-scale neuronal integration, can influence every cognitive act an agent might eventually perform. In studying these temporal dynamics, Varela and collaborators opted to focus on the phase relationships of brain signals, mainly because these contain a great deal of information on the temporal structure of neural signals, particularly those relating to the underlying mechanism for brain integration. Other authors have emphasized the relationship between phase information and memory formation and retrieval (Li and Hopfield 1989, Izhikevich 1999).

In robotics, although there has been much work on neurally-inspired coupled oscillator based control of complex rhythmic motor behaviours, particularly locomotion (e.g. Ijspeert et al. 2005), to date there has been very little research on the wider issues of neuronal synchronisation and phase information in the generation of embodied cognitive behaviours. The study described next is the first attempt to investigate the neural dynamics of a simulated robotic agent engaged in minimally cognitive tasks while employing an evolved instance of the Kuramoto model of coupled oscillators (Kuramoto 1984) as its nervous system. These tasks are simple enough to allow detailed analysis and yet are complex enough to motivate some kind of cognitive interest. The work has dual aims: both to shed new light on the possible role of neuronal synchronisation and phase information in the generation of sensorimotor cognitive behaviours - for instance to investigate whether different degrees of synchronisation are appropriate in different circumstances and what role non-synchronised, transient dynamics might play, and to begin investigating the efficacy of such systems as practical robotic controllers.

The first task is an active categorical perception problem (Beer 2003, Dale and Husbands 2010) in which the robot has to discriminate between moving circles and squares, as first introduced by Beer (2003). In the second task, the robotic agent has to approach moving circles with both normal and inverted vision, adapting to both conditions. Even though these tasks don't strictly require a network of coupled oscillators to be solved, they have been chosen because they are useful benchmarks in the evolutionary robotics and adaptive behaviour communities (DiPaolo 2000, Izquierdo 2006) and act as a suitable focus for the possible roles of synchronisation in a deliberately non-rhythmic behaviour with some relevance to cognition.

The rationale behind the choice of the Kuramoto model is that it describes the phase evolution of a set of connected oscillators and with some adjustments can be associated with groups of neurons firing at a periodic rate (Cumin and Unsworth 2007). Therefore, instead of focusing on single neuron activations, the model resembles the behaviour of groups of neurons. By using the phase dynamics as the central feature of the model, the emphasis is on short-term temporal activity, which has been previously shown to be successful in pattern recognition tasks (Tononi et al. 1992). Moreover, the model allows for easy inspection of the phase and frequency of each of the elements, which makes it especially suitable for studying synchronisation of groups of oscillators (Acenbron et al. 2005), a key factor when analysing communication and information processing in neuronal assemblies (Von der Marslburg 1981, Friston 2000). Izhikevich (1999) shows that depending on changes in phase relationships caused by external/internal stimulus, neurons can reorganize and synchronise themselves with different neurons, thus changing their response without the need to change synaptic weights. This points towards new kinds of behaviour generating mechanisms that are explored in the study described here. 


\section{The Kuramoto Model}

The Kuramoto model consists of a lattice of oscillators coupled according to Equation 7.

$$
\frac{d \theta_{i}}{d t}=\omega_{i}+\frac{k}{n} \sum_{j=1}^{n} \sin \left(\theta_{j}-\theta_{i}\right)
$$

where: $\theta_{\mathrm{i}}$ is the phase of the $i$ th oscillator, $\omega_{\mathrm{i}}$ is the natural frequency of the ith oscillator, $\mathrm{k}$ is the coupling factor between nodes and $\mathrm{n}$ is the total number of oscillators. If the frequency of any two given nodes $i$ and $j$ are equal, i.e. $d \theta_{i}-d \theta_{j}=0$ or $\theta_{i}-\theta_{j}=$ constant, the model is said to be globally synchronised.

It is possible to define a synchronisation index, which calculates how synchronised the set of oscillators are (Kuramoto 1984). A commonly used measure is defined in Equation 8, where $r$ is the synchronisation index (a value of 1 indicating high synchronisation, 0 indicating incoherent oscillatory behaviour) and $\psi$ is the mean phase of the system.

$$
r e^{i \psi}=\frac{1}{n} \sum_{j=1}^{n} e^{i \theta_{j}}
$$

The Kuramoto model has a set of properties that makes it suitable for the study of different types of synchronisation problems. The work described here focuses on a particular property known as partial synchronism. This phenomenon is exhibited when, in a globally synchronised network, changing the frequency of one node results in some of the nodes become synchronised while other nodes are not (Monteiro et al. 2003). Moreover, the oscillatory behaviour of one node can be influenced by another node in the network not necessarily connected to it. The importance of this property in mimicking brain related dynamics relies on the fact that different neuronal blocks could synchronise and influence other blocks (e.g. different cortical areas could flexibly establish communication channels depending on their temporal activity). This is in agreement with some recent findings in neuroscience (Buzsaki 2006), reinforcing the feasibility of applying the Kuramoto model to study cognitive processes.

\section{Experimental framework}

The Kuramoto model was modified so that it could be used to control a simulated robotic agent. The core of the robot controller is a set of oscillators, connected in two possible ways: to its immediately two neighbours, producing a ring structure (see Figure 5), or fully connected. In his original work, Kuramoto suggested a fully connected set-up, but other structures, including the ring shaped one, have been studied and proven to have a direct influence over the synchronisation properties of the model (Wiley et al. 2006, Cumin and Unsworth 2007, El-Nashar and Cerdeira 2009). 
The frequency of each node is calculated as the sum of its natural frequency, $\omega_{\mathrm{i}}$, and the value of the sensory input related to that node scaled by a factor $z_{\mathrm{i}}$. At each iteration the phase differences from a given node in relation to the set all other nodes it is connected to $\left(\mathrm{C}_{\mathrm{i}}\right)$ are calculated according to Equation 9 . Based on the approach suggested by Schmidhuber et al. (2007), the output of the network is given by the sine of the phase differences linearly combined by an output weight matrix W (Figure 5). The sine function smooths out phase difference instabilities caused by phases resetting when they exceeds $2 \pi$. Therefore there are $n$ inputs to $n$ corresponding nodes in the network, with $C_{n, 2}$ resulting phase differences and $o$ outputs created via a $\mathrm{C}_{\mathrm{n}, 2} \times o$ matrix $W$.

$$
\frac{d \theta_{i}}{d t}=\left(w_{i}+z_{i} I(t)\right)+k \sum_{j \in C_{i}} \sin \left(\theta_{j}-\theta_{i}\right)
$$

In this way, the overall behaviour of the network will be dictated by the phase dynamics and the environmental input to the robotic agent. It is important to stress that nodes that are not directly connected can still influence each other, depending on their frequencies.

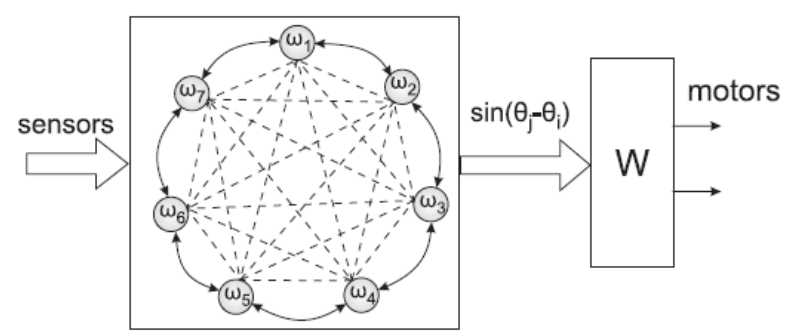

Figure 5: Framework for application in evolutionary robotics. The oscillatory network is represented by a set of nodes connected by a thick line, in the case of the ring topology, or by dashed lines, in the case of the fully connected topology.

The first experiment involved an active categorical perception task performed by a simulated circular robotic agent able to move horizontally at the bottom of a $250 \times 200$ rectangular environment (Figure 6). The robotic agent has 7 ray sensors, symmetrically displaced in relation to the central ray in intervals of $\pm \pi / 12$ radians, and two motors. An intersection between a sensory ray and an object gives a sensor reading between 0 and 10 , 0 when the ray length is greater than 200 units and 10 when the ray length is 0 . In all experiments, sensors are saturated (they are clamped) when their value is above 9 . The robotic agent has to discriminate between circles and squares as they fall from the top of the arena to the bottom (only one object at each trial), where the robotic agent is located. The square's diagonal, the robotic agent's and the circle's radius all measure 15 units. At the beginning of each trial, a circle or a square is dropped from the top of the environment in a random horizontal position within a maximum of 50 units from the robotic agent, and moves vertically with a velocity of 3 units/step. The robotic agent, constrained to move (left and right) in a straight line along the bottom, has to approach the circles and avoid the squares, adjusting its horizontal velocity accordingly. 
The second experiment consists of an orientation task. In the same environmental set-up, the robotic agent has to adjust its horizontal position to catch falling circles, with both normal and inverted vision. When submitted to visual inversion, sensory readings from an object at the right side of the agent are perceived by the agent's left set of sensors, and vice-versa. Therefore, different scenarios can cause similar or identical sensory stimulus to

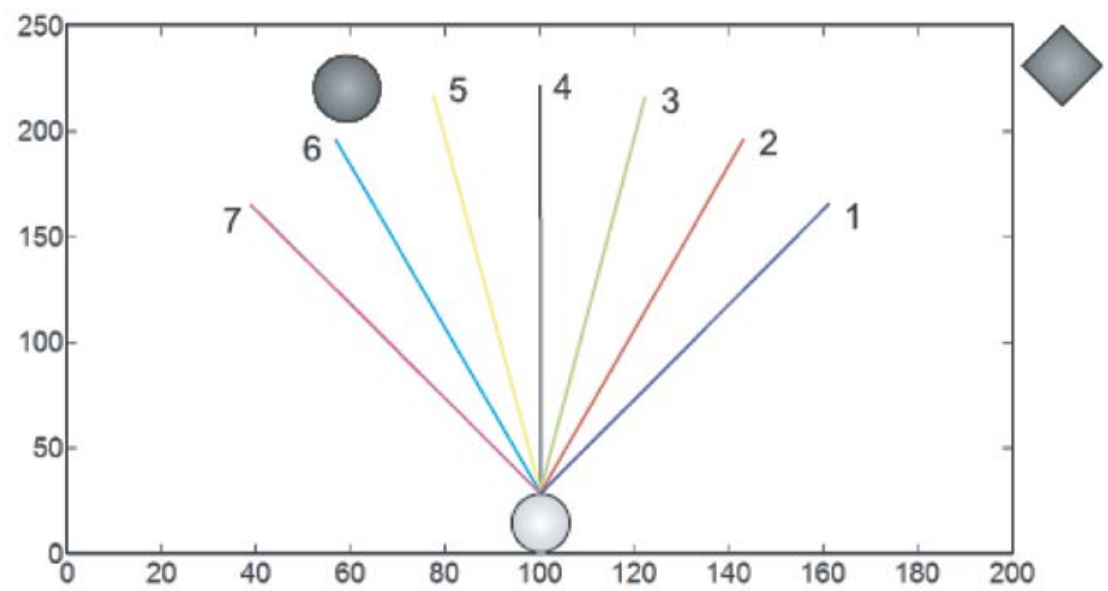

Figure 6: Experimental scenario. The agent (gray circle at the bottom) has to catch falling circles and avoid squares in Task 1 and catch falling circles with normal and inverted vision in Task 2 . The robotic agent has 7 ray sensors, symmetrically displaced with relation to the central ray in intervals of $\pm \_/ 12$ radians, and two motors that can move it horizontally. The numbers next to the sensors show the correspondence between the agent's sensory input and the nodes of the network.

the robotic agent; the agent is required to develop a strategy that can overcome the sensory disruption.

A genetic algorithm is used to determine the parameters of the system: the frequency of each node, $\omega_{i} \in[0,10]$, the coupling factor $k \in[0,5]$, the input weights $\mathrm{z}_{\mathrm{i}} \in[0,3]$, the matrix $\mathrm{W}$ with elements in the interval $[-2,2]$ and motor output weight $\mathrm{s} \in[0,10]$, resulting in a genotype of length 58 for the tasks studied here. The network's genotype consists of an array of integer variables lying in the range $[0,999]$ (each variable occupies a gene locus), which are mapped to values determined by the range of their respective parameters. For all the experiments reported here, a distributed GA similar to that described in the GasNets section above (and in Husbands et al. 1998) was used; the population size was 49 , arranged in a $7 \times 7$ grid. A generation is defined as 49 breeding events and the evolutionary algorithm runs for a maximum of 150 generations. Two mutation operators were used: the first operator was applied to $20 \%$ of the gene and produces a change at each locus by an amount within the $[-10,+10]$ range according to a uniform distribution. The second, more disruptive, mutation operator was applied with a probability of $10 \%$ and is applied to $40 \%$ of the genotype. A randomly chosen gene locus is replaced with a new value within the $[0,999]$ range in a uniform distribution.

In the first experiment, fitness is evaluated over a set of 28 trials with randomly chosen objects (circles or squares), starting at a uniformly distributed horizontal offset in the interval of \pm 50 units from the robotic agent. Fitness is defined as the robotic agent's ability to catch circles and avoid squares, and is calculated according to the following 
function: fitness $=\sum_{i=1}^{N} i f_{i} / \sum_{i=1}^{N} i$ where $f_{i}$ is the ith value in a descending ordered vector of evaluation scores for separate trials, and is given by $1-\mathrm{d}_{\mathrm{i}}$, in the case of a circle, or by $\mathrm{d}_{\mathrm{i}}$ in the case of a square where $d_{i}$ is the (normalised) horizontal distance from the robotic agent to the object at the end of the ith trial (when the object reaches the bottom of the environment). Therefore, a robotic agent with good fitness maximizes its distance from squares and minimizes its distance from circles. Note that the form of the fitness function is the same as that of Equation 6, providing strong pressure for good performance in all trials (generalisation).

In the second experiment, fitness is evaluated over a set of 20 trials with normal vision followed by 20 trials with inverted vision. The circles are dropped at an uniformly distributed horizontal offset in the interval of \pm 50 units from the robotic agent. Fitness for each part of the run is defined as above but considering just the circle catching scenario. The final fitness is calculated by averaging the fitness obtained under normal and inverted vision.

\section{Results}

In the first experiment (catch circles, avoid squares) two network topologies were investigated, a ring topology and a fully connected topology. Both architectures produced similarly good results, providing an existence proof that oscillator phase dynamics can be useful, as part of a situated embodied system, in driving autonomous sensorimotor behaviour. The training fitness of the best ring topology individual was 0.96 out of 1.00 , and the generalisation fitness over 100 random runs was 0.94, which is comparable with the results that are found in the literature (Beer 2003, DiPaolo 2000). For the fully connected topology the training fitness of the best evolved individual was 0.97 , and the generalisation fitness over 100 runs was 0.92 . Analysis of successful individuals reveals clearly different dynamics are at play in circle catching and square avoidance; this is true for both styles of network architecture. Further, although synchronised states play an important role, unsynchronised and transient dynamics are also significant (Moioili et al. 2010, 2012, Santos et al. 2012). Figure 7 shows the synchronisation index plotted against time for the best individuals from experiment one. This clearly shows that the phase dynamics are different for circle catching and square avoidance for both architectures. Square avoidance mainly exploits synchronised dynamics ( $\mathrm{r}$ for square catching can be seen to always remain very close to 1 ) whereas circle catching makes significant use of unsynchronised dynamics ( $\mathrm{r}$ for circle catching deviates significantly from 1 after about 40 iterations).

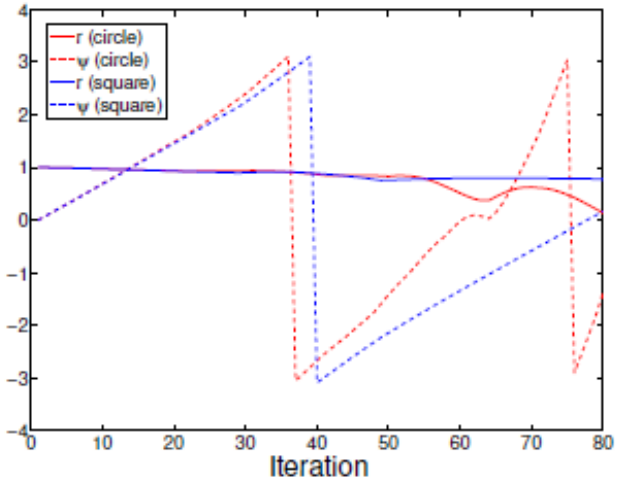

(a)

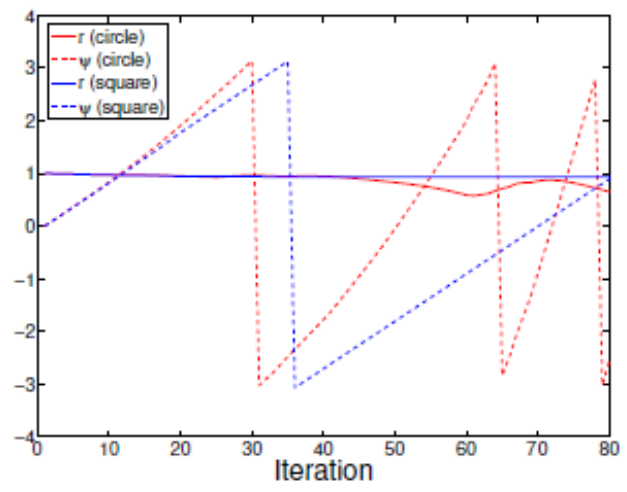

(b) 
Figure 7: Synchronisation index (r) and the mean phase $(\psi)$ of the network for the ring topology (a) and the fully connected topology (b), calculated according to Equation 8 for best individual evolved in the first experiment.

In the second experiment (catching falling circles under normal and inverted vision), the robotic agent was controlled by the fully connected network architecture, given its slightly better performance obtained in the previous experiment in the catching circles part of the task. Again agents with very good performance were successfully evolved. The training fitness for the best evolved individual was 0.94 out of 1.00 , and the generalisation fitness over 100 random trails was 0.93 . Generalisation performance is illustrated in Figure 8. Under both conditions, the main adopted strategy seemed to be: move to one side of the object (in this case the left side), where robotic agents with normal visual have their right sensors stimulated whereas robotic agents with inverted vision have their left sensors stimulated, and then centre in on the object.

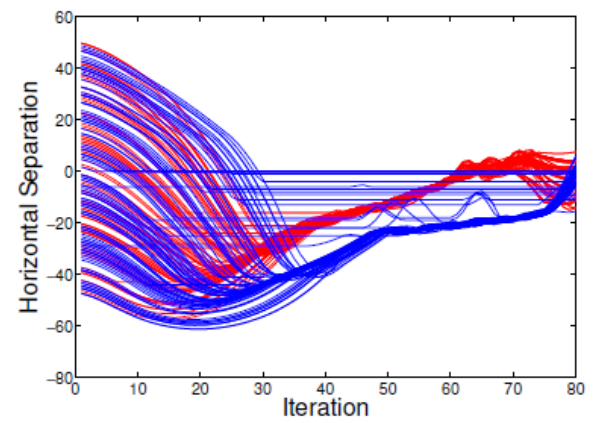

Fig. 8. The generalisation performance of the agent over 100 random trails in Experiment 2 . The red colour is related to the normal vision case and the blue colour to the inverted vision scenario. The plot illustrates the value of the horizontal separation of the agent and the object over 80 iterations

Looking at Figure 9 it is possible to see that although the strategy for the normal and inverted vision tasks is almost the same, the oscillatory activity of the network and its phase dynamics are quite different, illustrating the multiple roles a single oscillator can have in the network. For example, near iteration 60, under the normal vision condition (upper part of the figure, middle graphic), the frequency of each node is varying and there is no apparent synchronisation. Near the same iteration, for the inverted vision case (bottom part of the figure), one can observe two almost synchronised clusters appearing: one formed by 4 nodes, the other formed by 2 nodes and the unsynchronised remaining node oscillating at a much higher frequency. This demonstrates an interesting adaptive mechanism that does not require changes in synaptic strengths but rather works by changing phase and degree of synchronisation within neural subgroups that form, break apart and reconfigure throughout the duration of the behaviour. This is another example of the 'shifting network' (Husbands et al. 2001) where reconfiguring network dynamics underlies plasticity of behaviour. 

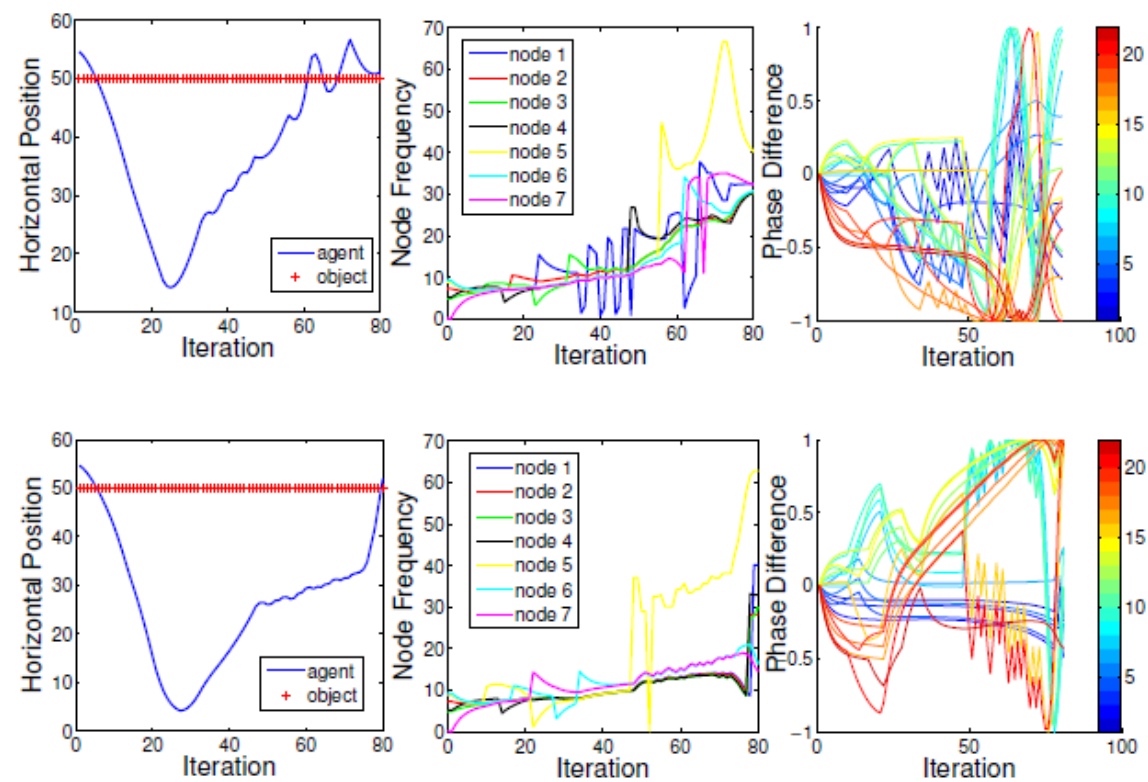

Fig. 9. Detailed behaviour of the agent's internal and external dynamics in Experiment 2. The left column illustrates the horizontal coordinate of the agent and the object, the middle one shows the frequency of each node of the network as the task progresses and the right one presents the phase differences.

This analysis again illustrates that evolution is able to readily exploit the rich dynamics the networks are capable of and, importantly, does not rely solely on simple synchronised states in behaviour generation. This work is an initial step along a path that could eventually provide insights into the role of synchronised (and unsynchronised) neuronal dynamics in the generation of cognitive and sensorimotor behaviours. For an extended discussion of related work see (Moioli et al. 2012, Santos et al. 2012).

\subsection{Exploiting Chaotic Dynamics in an Embodied System}

This section describes research that is not strictly evolutionary robotics but is closely related and highlights some important emerging topics that are highly relevant to ER and its intersection with neuroscience.

The possibility of exploiting intrinsic chaotic dynamics has recently attracted the attention of both neurobiologists interested in how animals learn to coordinate their limbs (Kelso 1995, Korn and Faure 2003, Mpitsos et al. 1988), for instance in locomotion behaviors, and roboticists striving to develop better, more efficient locomotion systems for articulated autonomous robots (Kuniyoshi and Suzuki 2004, Pitti et al. 2010, Steingrube et al. 2010). Chaotic dynamics emerging spontaneously from interactions between neural circuitry, bodies and environments can be used to power a kind of search process as an embodied system explores its own possible motor behaviors. However, to date it has not been clear how to harness chaos in a general goal-direct way such that desired adaptive sensorimotor behaviors can be explored, captured and learnt. In this section we briefly present a general and fully dynamic embodied neural system, which exploits chaotic search through adaptive bifurcation, for the real-time goal-directed exploration and learning of the 
possible locomotion patterns of an articulated robot of an arbitrary morphology in an unknown environment. Our results show that the novel neuro-robotic system is able to create and learn a number of emergent locomotion behaviors for a wide range of body configurations and physical environments, and can re-adapt in real-time after sustaining damage. For further details of the methods see Shim and Husbands (2010, 2012).

Properly coordinated rhythmic movements for locomotion are ubiquitous in animals. Biological locomotor systems (usually involving coordinated limb movements) evolved to be highly adaptable, dexterous and energy efficient. Consequently they are a major source of inspiration when designing robot locomotion systems. Most biological locomotor systems involve neural networks acting as central pattern generators (CPGs) which are responsible for producing the basic rhythmic patterns for the oscillatory movement of limbs. Understanding the subtleties of operation of such networks, and how to design artificial versions for robotic applications, are on-going challenges.

Most approaches to designing CPG-based robotic locomotor systems have relied on optimization and search methods, including evolutionary algorithms and other stochastic methods, to find a suitable configuration of system variables - including the ER approaches to locomotion described earlier. These methods can be computationally expensive and often require a priori knowledge of the robot body and environment. Hence there are still many open issues in how to deal with unknown environments and adaptation to arbitrary or changed (e.g. damaged) body conditions in the most general and efficient way.

In robotics, a greater appreciation of the importance of framing behavior in terms of brainbody-environment interactions has led to efforts to exploit various ready-made functionalities provided by the physical properties of an embodied system. A recent strand of work has built on the growing body of observations of intrinsic chaotic dynamics in nervous systems to suggest that such dynamics can underpin crucial periods in animal development when brain-body-environment dynamics are explored in a spontaneous way as part of the process of acquiring motor skills. Recent robotics studies have demonstrated that chaotic neural networks can indeed power the self-exploration of brain-bodyenvironment dynamics in an embodied system, discovering stable patterns that can be incorporated into motor behaviors (Pitti et al 2010, Kuniyoshi and Sangawa 2006). In the work outlined here we significantly generalize and extend this previous research to demonstrate how chaotic neural dynamics can be harnessed to develop a kind of system not seen in previous models: one where intrinsic neural dynamics can be used to autonomously explore, capture and learn whole goal-directed sensorimotor behaviors in an embodied system without recourse to external monitoring, evaluation or training methods. We introduce a general on-line and fully dynamic neural process for the exploration and learning of possible locomotion patterns for articulated robots of an arbitrary morphology in unknown physical environments. Goal directed exploration is achieved using chaotic search while discovered patterns are memorized and sustained by adaptive changes to the wirings of chaotic neural oscillators that form the basis of the neural architecture. As well as having direct application in robotics, this work has potential implications for neurobiology. 
Conventional optimization and search strategies generally use random perturbations of the system variables to search the space of possible solutions. We have developed a method which uses the intrinsic chaotic dynamics of the system to naturally power a search process without the need for external sources of noise (Shim and Husbands, 2010, 2012). We employ the concept of Chaotic Mode Transition with external feedback (Davis, 1990), which exploits the intrinsic chaoticity of a system orbit as a perturbation force to explore multiple synchronised states of the system, and stabilises the orbit by decreasing its chaoticity according to a feedback signal that evaluates the behaviour. An evaluation signal which measures how well the locomotion behavior of the system matches the desired criteria (e.g. locomote as fast as possible) is used to control a bifurcation parameter which alters the choaticity of the system. During exploration, the bifurcation parameter continuously drives the system between stable and chaotic regimes. If the performance reaches the desired level, the bifurcation parameter decreases to zero and the system stabilizes. A learning process that acts in tandem with the chaotic exploration captures and memorizes these high performing motor patterns.

The overall architecture of the system is illustrated in Figure 10, the neural architecture generalizes and extends that presented in (Kuniyoshi and Sangawa 2006) which is inspired by the organization of spinobulbar units in the vertebrate spinal cord and Medulla Oblongata (the lower part of the brainstem which deals with autonomic, rhythmic, involuntary functions). Each joint in an articulated robot is connected to a motor unit comprising a pair of central pattern generator (CPG) neurons which receive sensory input. The neurons produce motor outputs for an antagonistic muscle pair that control the movement of the joint. Each CPG neuron is modeled as an extended Bonhöffer van der Pol (BVP) oscillator (FitzHugh 1961, Asai et al. 2003), which can be viewed as a simplification of the full Hodgkin Huxley neural model (Hodgkin and Huxley 1952). The CPG neurons are all connected to each other but these connections are initially made inactive. The CPG neurons receive sensory signals that integrate information from the body-environment interaction dynamics experienced by the system (e.g. from force and position/angle sensors). Hence, while the direct connections are inactive, any coupling between the oscillators will be indirect via bodily and environmental interactions. The network of oscillators, coupled through physical embodiment, has multiple synchronized states (modes) that reflect the body schema and its interactions with the environment, each of which can be regarded as a potential candidate for 'meaningful' motor behavior. The exploration process, powered by adaptive bifurcation through the feedback evaluation signal, allows the system to become entrained in these modes, one at a time, until one is found that is sufficiently stable and high performing for the bifurcation parameter to reduce to zero and the system to fully stabilize. As the system stabilizes, the connections between oscillators are dynamically activated using an adaptive synchronization learning scheme. In this way the wiring between the oscillators is changed in order to capture and maintain the high performing motor pattern. The learning rule is also controlled by the bifurcation parameter and is set up such that the connections between the oscillators are effectively zero (inactive) during the exploration process but gradually increase (become active) as the system nears stability (see Shim and Husbands 2012 for full details). Thus, exploration and learning are merged as a continuous dynamical process such that the desired motor behavior is spontaneously explored, discovered, and memorized in a coherent way. If the performance drops, for instance following a change in the environment or damage to the body, the system will automatically return to the exploration phase until a new stable high performing motor pattern is discovered. 
The pair of CPG neurons in each motor unit (labeled $l$ and $r$ ) operate according to the following coupled differential equations:

$$
\begin{gathered}
\tau \dot{x_{l}}=c\left(x_{l}-\frac{x_{l}^{3}}{3}-y_{l}+z_{1}\right)+\delta\left(H_{l}\left(s_{l}\right)-x_{l}\right)+F_{l}^{x} \\
\tau \dot{y_{l}}=\frac{1}{c}\left(x_{l}-b y_{l}+a\right)+\varepsilon\left(H_{l}\left(s_{l}\right)\right)+F_{l}^{y} \\
\tau \dot{x_{r}}=c\left(x_{r}-\frac{x_{r}^{3}}{3}-y_{r}+z_{2}\right)+\delta\left(H_{r}\left(s_{r}\right)-x_{r}\right)+F_{r}^{x} \\
\tau \dot{y_{r}}=\frac{1}{c}\left(x_{r}-b y_{r}+a\right)+\varepsilon\left(H_{r}\left(s_{r}\right)\right)+F_{r}^{y}
\end{gathered}
$$

Where $\tau$ is a time constant, and $a=0.7, b=0.675, c=1.75$ are the fixed parameters of the oscillator. Each consecutive pair in the set of $2 \mathrm{~N}$ oscillators are sequentially allocated to each motor unit as $l=2 \mathrm{~m}-1$ and $r=2 \mathrm{~m} . \delta=0.013$ and $\varepsilon=0.022$ are the coupling strengths for afferent input $H(s)$ which is a function of raw sensor output $s$, processed by the sensor adaptation module (SAM) - see Figure 10. $F_{i}^{j}$ is a coupling term between oscillators and is subject to the learning process. $z_{1}$ and $z_{2}$ are the control parameters for adjusting the chaoticity of the motor unit. Their difference $\left(\mu=z_{2}-z_{1}\right)$ changes identically in all motor units, and acts as the global bifurcation parameter. In the stable regime where the two control parameters are symmetric, it had been found (Asai et al. 2003) that the two coupled BVP equations exhibit bistable phase locking of their oscillations in a parameter range of $0.6<z_{1}=z_{2}<0.88$. From the observation of a number of experiments on the oscillator dynamics, we chose to fix $z_{2}=0.73$ and to vary $Z_{1}$ in order to ensure a higher probability of multistability of the system in its stable regime. For further details see (Shim and Husbands 2012).

The evaluation signal is determined by a ratio of the actual performance (e.g. forward speed) to the desired performance. Where the desired behavior is forward locomotion, the evaluation signal, $E$, is measured according to the following equations, where $v$ is the robot velocity vector and $\tau_{\mathrm{E}}$ is a time constant. Using this leaky integrator equation means the velocity is continuously averaged over a time window thus eradicating gyrations and oscillations.

$$
E(t)=\|\overline{\boldsymbol{v}}\|, \quad \tau_{E} \frac{d \overline{\boldsymbol{v}}}{d t}=-\overline{\boldsymbol{v}}+\boldsymbol{v}
$$

The time course of the bifurcation parameter, $\mu$ (Equation $\mathrm{XX}$ ), is tied to the evaluation signal using the following equations.

$$
\tau_{\mu} \frac{d \mu}{d t}=-\mu+\mu_{c} G\left(\frac{E}{E_{d}}\right), \quad G(x)=\frac{1}{\left(1+e^{16 x-8}\right)}
$$

Where $\tau_{\mu}$ and $\mu_{\mathrm{c}}$ are constants and $\mathrm{E}_{\mathrm{d}}$ is the desired performance; $\mathrm{G}(\mathrm{x})$ implements a decreasing sigmoid function which maps monotonically from $(0,1)$ to $(1,0)$ shaped so that the boundary value at $\mathrm{x}=1$ and its derivative become almost 0 so as to make the function smoothly vanish to zero to facilitate gradual stabilisation. Since the method is intended for 
use in the most general case, where the robotic system is arbitrary, we do not have prior knowledge of what level of performance it can achieve. Using the concept of a goal setting strategy (Barlas and Yasarcan 2006), the dynamics of the desired performance are modeled as a temporal average of the actual performance, such that the expectation of a desired goal is influenced by the history of the actual performance experienced as described by the following equation.

$$
\tau_{d} \frac{d E_{d}}{d t}=-E_{d}+E
$$

The sensor signal fed to a CPG neuron undergoes homeostatic adaptation as it passes through a sensor adaptation module (SAM) before reaching the neuron (see Figure 10). The SAMs were introduced because by adjusting the waveforms of input signals to be close to those of the neural activities, the synchronicity between the neural and physical system was enhanced thus allowing the neural system to cope with an arbitrary robotic system. This regulation also results in a diversification of output behaviors, increasing the scale of the search process.

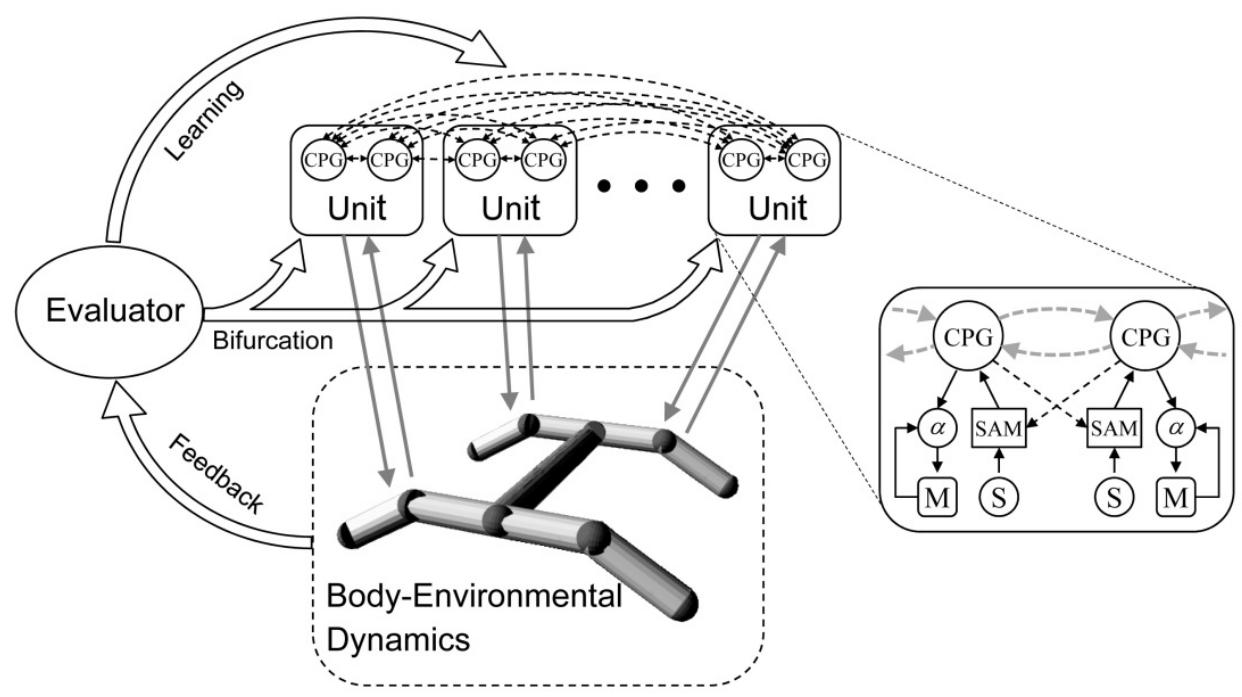

Figure 10: An overview of the integrated exploration and learning scheme. Each robot joint has a dedicated motor unit comprising oscillator- based central pattern generator neurons (CPG) with sensory input (S) and motor output $(\mathrm{M})$. Connections between the oscillators are initially inactive but they are weakly coupled through the body and environment. An evaluation feedback signal controls a global bifurcation parameter that alters the chaoticity of the CPGs. The chaotic dynamics of the neutron-body-environment system drive a search process that finds motor patterns that perform well according to the evaluation criteria. As the system stabilizes on a high performing pattern the bifurcation parameter reduces to zero and the connections between the oscillators become active, their weights being set by a learning procedure that is smoothly linked to the chaotic exploration process. The learning process further stabilizes, captures and memorizes the motor patterns. Sensory input undergoes homeostatic adaptation as it passes through a sensor adaptation module (SAM). This enhances the synchronicity between the neural and physical system thus allowing the neural system to cope with an arbitrary robotic system. 
The chaotic exploration and learning system was evaluated by using it to control a range of realistically simulated articulated robots that were required to locomote in an effective way. Successful experiments with a swimming robot and a variety of walking robots of differing morphologies demonstrated that the framework is highly general (Shim and Husbands 2012). In each case a range of stable locomotion behaviors were discovered and learnt. It was also shown that the robots can readily readapt after damage or other changes.

The seamless interaction between the exploration and learning processes results in a system that can be thought of as continually self-monitoring in order to maintain an appropriate level of motor function. As well as being an effective means of developing robotic controllers, the method has more general implications for truly autonomous artificial systems which must maintain their integrity on several levels, including behavioral. Because of its strong biological inspiration it also serves as an indication of the kinds of processes that may be operating in natural nervous systems.

Figure 11 shows two of the robot simulations used to demonstrate the system. Figure 12 shows a typical swimming motion discovered and captured for the 4-Fin swimmer. It is an efficient frog-like motion. Figure 13 illustrates how the method allows real-time adaptation. The robot suffers damage where the length of the fin on arm 4 was reduced by $90 \%$. Its performance reduced almost immediately to zero so the exploration process automatically kicked-in. Very quickly it discovers and stabilises one of the high performing transient patterns, needing only a few trials for the oscillator learning mechanism to completely stabilise the system once more into a new high performance swimming motion that is able to compensate for the damage.
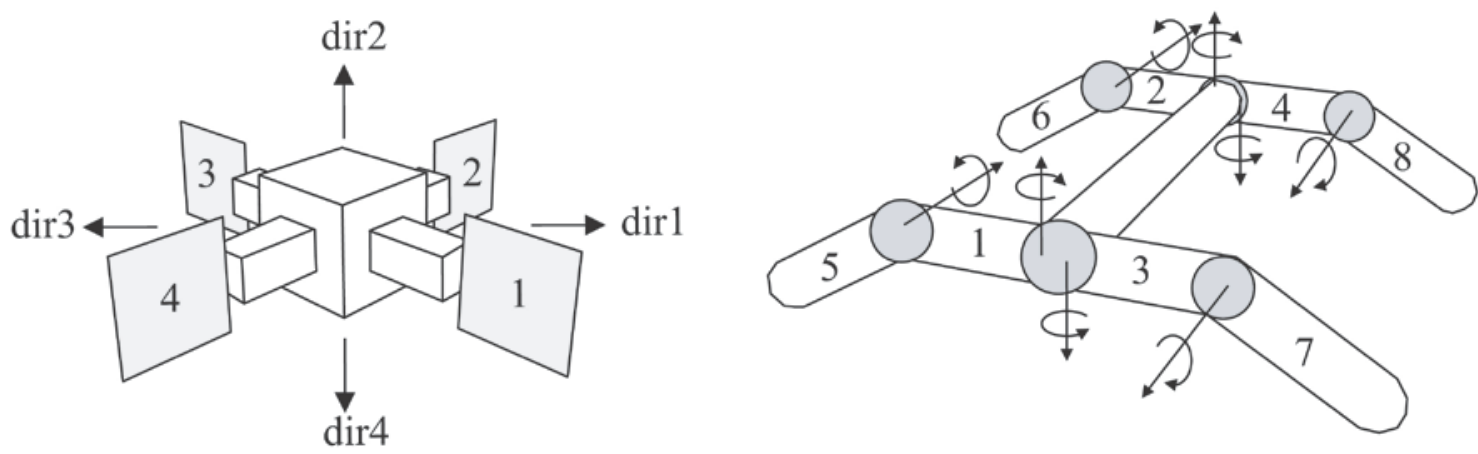

Figure 11: Robotic simulation models of a 4-Fin Swimmer (2D movement) and a Quadruped (3D movement) used to evaluate the method. 


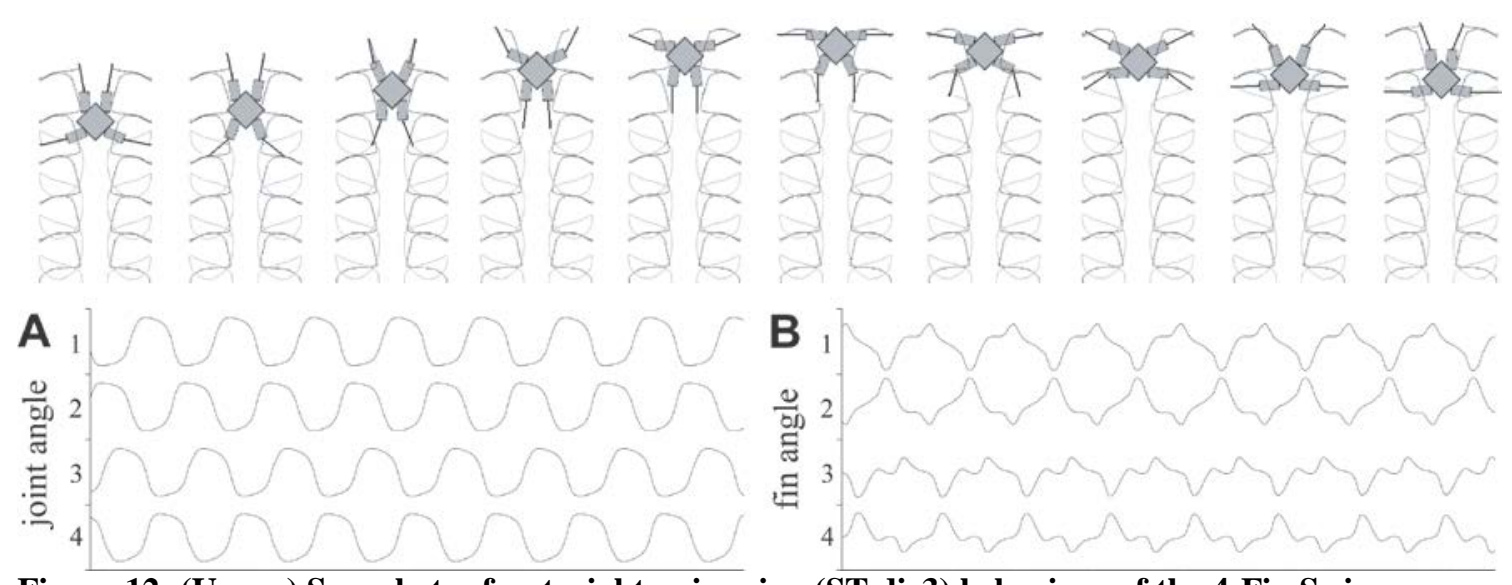

Figure 12: (Upper) Snapshots of a straight swimming (ST dir3) behaviour of the 4-Fin Swimmer developed by the exploration and capture method. Images were taken every 1/10 gait cycle. The tip trajectories of the fore (fin 3,4:black) and rear (fin1,2:grey) fins are shown. (Lower) (A) Joint angles and $(B)$ fin bending angles of the behaviour. Each segment along the vertical axis indicates the range $[-1,1]$ rad.
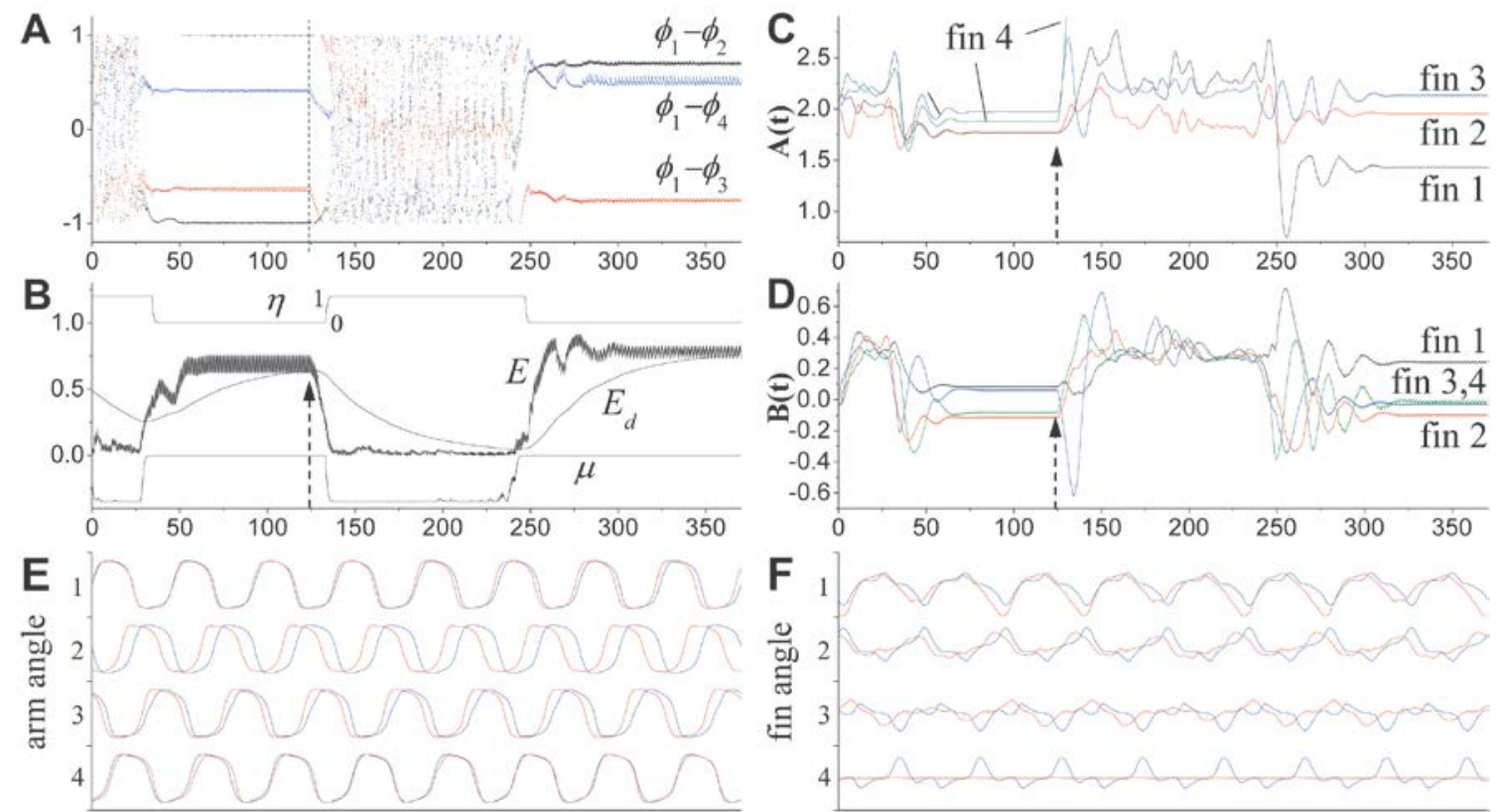

Figure 13: Realtime recovery after a radical change to the body of the swimmer (damage). Dashed lines and arrows indicates the time of damage, when the length of fin 4 is decreased to 1/10 of its original length. The sensor gain of (damaged) fin $4(A(t) \approx 5.0)$ in $(C)$ was truncated for a better view of the other gain plots. (E) and (F) show the joint angles and the fin angles respectively, where the undamaged motion (blue) and the readapted motion (red) are superposed. The fiducial point for the superposed plots was set to the starting point of arm angle 1 in (E).

Figure 14 illustrates the generality of the method by showing how it quickly discovers, captures and stabilises high performing walking behaviour in a quadruped robot model. For details of applications of the method to other robots and body morphologies see Shim and Husbands (2012). 

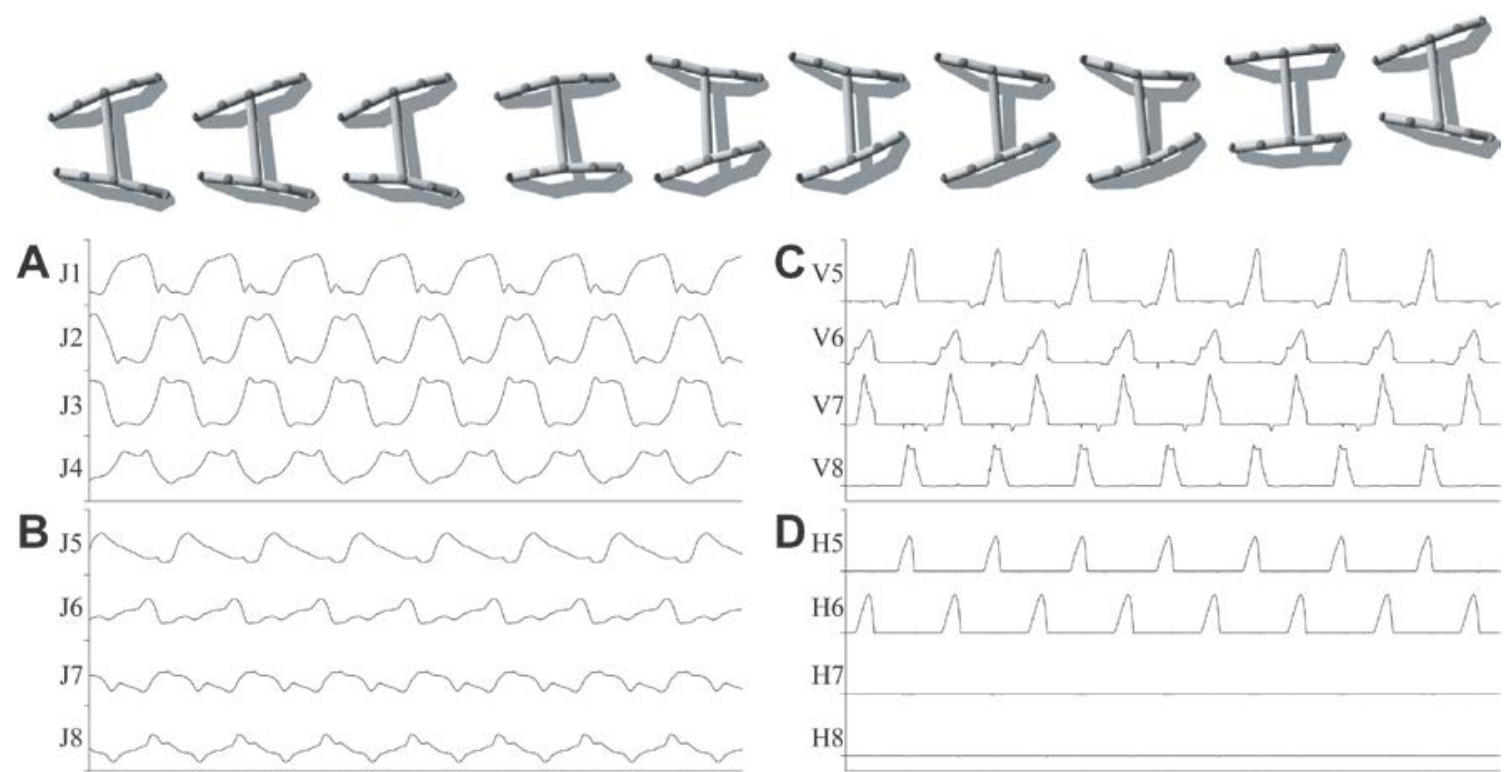

Figure 14: An example of a quadruped gait captured by the exploration-learning process. Snapshots were taken every 1/10 gait cycle. (A,B) The joint angles of limbs. (C) The horizontal speeds of each foot (the tips of limbs 5-8) in the direction of locomotion. (D) The height of each foot from the ground. The two rear feet $(\mathrm{V7,V8,H7,H8)} \mathrm{show} \mathrm{stick-and-slip} \mathrm{movements} \mathrm{on} \mathrm{the} \mathrm{ground} \mathrm{under}$ Coulomb friction. The range of each plot is as follows; J1-J8: [-1.0,1.0]rad, V5-V8: [0.0,2.0]m/s, H5H8: $[0.0,0.08] \mathrm{m}$.

Just as incorporating various forms of neural plasticity into ER has proved very fruitful (Floreano and Urzelai 2000, Husbands et al. 1998, DiPaolo 2003), the integration of chaotic dynamics, as outlined in this section, may result in a powerful hybrid method. There are a number of parameters in the chaotic method that need to be set by hand. ER methods may be able find better setting for combinations of these parameters in order to further increase the efficiency of the method. But a potentially more powerful hybridisation of the chaotic search and learning method with ER will be to allow the exploration of more complex architectures within an evolutionary framework. This may involve the integration of spinobulbar motor units within a more complex overall architecture, for instance to allow a variety of behaviours, or the development of alternative architectures for the motor units themselves.

\section{Discussion and Prospects}

Although great progress has been made in autonomous robotics over the past few decades, and techniques similar to those discussed in this chapter have played their part in some of the ever-proliferating mobile robots we now see in the home (e.g. autonomous vacuum cleaners and toys), or in areas such as planetary exploration, security or military applications, many challenges remain.

It is now possible to produce autonomous robots that behave in a robust and reliable way in real environments, engaging in real tasks in real-time. However, the behaviours involved are still relatively simple. Progress has been slow towards more sophisticated tasks such as learning what to focus attention on in a complex environment, coordinating many conflicting goals and drives, interacting with other robots in complex group 
behaviours, learning to communicate in a sophisticated way about the task at hand, and so on. Perhaps this should not be at all surprising. One lesson that most neuroscientists have understood for many decades, but which has often been overlooked in AI, is that the generation of intelligent embodied behaviour is an extremely complicated problem. However, progress is being made and there are many promising lines of research. It is likely that directions involving artificial neural systems and other biologically inspired methods will become even more important as attempts to tackle these hard problems gather momentum. ER methods will surely play a role, either as a standalone direction or in concert with other approaches. One direction, mentioned in several examples described earlier, that is likely to become increasingly important is the continued dismantling of the line between brain and body that has traditionally been present in studies of both natural and artificial intelligence. The tighter integration of artificial bodies and brains at many different levels, as suggested in Pollack's chapter in this book (Pollack 2012), is an intriguing possibility that would probably require advances in evolutionary developmental systems - something that could usher in an exciting new direction in ER research.

There are a number of potentially important emerging fields that may have a radical impact in the decades to come. These include developments in interfacing digital electronics to neural tissue. The most frequent motivation for such work is to allow improved prosthetics to be directly controlled by the nervous system. This points to the possibility of an increased merging of robotic technology with human bodies something that a number of people have reflected on recently (e.g. Brooks 2002) and which the work of Stelarc, the radical performance artist, has long explored (Smith 2007). A related area involves attempting to harness the sophisticated non-linear adaptive properties of cultured (real) neural networks to create hybrid machines (DeMarse et al. 2001), pointing towards the possibility of robots that include biological matter in their control systems - a development that would echo the imagined landscapes of dozens of sci-fi books and movies. It is possible that in the long-run that kind of approach may prove more powerful than attempting to understand biological systems in sufficient detail to be able to abstract general mechanisms underlying the generation of intelligent behaviour. However, such research is at an extremely early stage, so we cannot yet properly assess its potential. However, one approach that has been considered recently is the use of artificial selection to shape cultured networks towards some behaviour end (Bull 2004). This is very difficult with current technology, but advances in high density multi-electrode array hardware, allied with chemical perfusion systems, might allow sufficiently powerful and repeatable manipulation of neural networks to make this a viable approach.

There is little doubt that many powerful biological neural mechanisms have not yet been discovered. One intriguing hypothesis is that one of the forms of plasticity on which the brain relies is itself a form of evolution via natural selection acting within neural tissue (Fernando et al. 2008, Fernando et al. 2011). Irrespective of whether or not it occurs in nature, this kind of mechanism could be employed in a whole new kind of evolutionary robotics.

The field of robotics has massively expanded since the days when it was dominated by cumbersome industrial arms; it is now quite possible that in the not too distant future robots will become as widespread and as common as computers are now. If such a technological revolution comes to pass, it is highly likely that artificial neural systems 
will play an important part as there will be greater demands for robust, reliable adaptation and learning, as well as sophisticated pattern recognition and sensory processing - all areas in which neural systems have great potential, especially within the context of behaving embodied systems. This in turn means that there are great opportunities for the kinds of interactions between ER and neuroscience described in this chapter.

\section{References}

AB (2009) Adaptive Behavior 17(4). Special issue on robotic and agent modelling of adaptive behaviour, based around responses to Webb (2009).

Abbott, L. F. and Kepler, T. B. (1990). Model neurons: from Hodgkin-Huxley to Hopfield. In Garrido, L., editor, Statistical Mechanics of Neural Networks. pp.5-18, Springer, Berlin.

Acebron, J., Bonilla, L., PerezVicente, C., Ritort, F. and Spigler, R. (2005) The Kuramoto model: A simple paradigm for synchronization phenomena. Reviews of Modern Physics, 77(1):137-185.

Asai Y, Nomura T, Sato S, Tamaki A, Matsuo Y, Mizukura I, Abe K (2003) A Coupled Oscillator Model of Disordered Interlimb Coordination in Patients with Parkinson's Disease. Biol Cybern 88:152-162.

Baranano, D., Ferris, C., \& Snyder,S. (2001) Atypical neural messengers. Trends in Neuroscience, 24(2), 99-106.

Barlas Y, Yasarcan H (2006) Goal Setting, Evaluation, Learning and Revision: A Dynamic Modeling Approach. Eval Program Plann 29(1):79-87.

Barnett, L.C., and Seth, A.K. (2011). Behaviour of Granger causality under filtering: Theoretical invariance and practical application, Journal of Neuroscience Methods. 201:404-419

R.D. Beer, H.J. Chiel, and L.S. Sterling. (1989) Heterogeneous neural networks for adaptive behavior in dynamic environments. In D. Touretzky, (Ed), Neural Information Processing Systems 1, pages 577-585, Morgan Kauffman.

Beer, R. and Gallagher, J. (1992) Evolving dynamical neural networks for adaptive behaviour. Adaptive Behaviour, 1:94-110.

Beer, R. (1995) A dynamical systems perspective on environment agent interactions Artif. Intell. 72:173-215.

Beer, R.D., Quinn, R.D., Chiel, H.J., \& Ritzmann, R.E. (1997). Biologically-inspired approaches to robotics. Communications of the ACM, 40(3), 30-38. 
Beer, R. (2003) The dynamics of active categorical perception in an evolved model agent. Adaptive Behavior, 11(4):209-243.

Berger, H. (1929) Uber das elektrenkephalogramm des menschen. Arch. Psychiat. Nervenkr., 87:527-570.

Brooks, R. (1991) Intelligence without representation. Artif. Intell. 47:139-159.

Brooks, R.A. (2002) Flesh and Machines: How Robots Will Change Us, New York: Pantheon Books.

Bull, Larry (2004). Personal communication.

Bullock, T., Bennett, M., Johnston, D., Josephson, R., Marder, E., \& Fields, R. (2005) The neuron doctrine, redux. Science, 310(5749), 791.

Buzsaki, G. (2006) Rhythms of the Brain. Oxford University Press.

Clark, A. (1999) An embodied cognitive science? Trends in Cog. Sci. 3(9):345-351.

Cliff, D., Harvey,I. and Husbands, P. (1993) Explorations in evolutionary robotics. Adaptive Behavior, 2:73-110.

Collins, R., Jefferson, D. (1991) Selection in massively parallel genetic algorithms. In R. Belew (ed), Proc. 4th Int. Conf. GAs, Morgan Kaufmann, 249-256.

Cumin, D. and Unsworth, C. (2007) Generalising the Kuramoto model for the study of neuronal synchronisation in the brain. Physica D, 226(2):181-196.

Dale, K. and Husbands, P. (2010) The Evolution of Reaction-diffusion Controllers for Minimally Cognitive Agents, Artificial Life 16(1):1-19.

Davis, P. (1990). Application of optical chaos to temporal pattem search in a nonlinear optical resonator. Japanese Journal of Applied Physics, 29, L1238-L1240.

Davis, G. (2006) Homeostatic Control of Neural Activity: From Phenomenology to Molecular Design, Annual Review of Neuroscience 29: 307-323.

Dawson, T., \& Snyder, S. (1994) Gases as biological messengers: nitric oxide and carbon monoxide in the brain. Journal of Neuroscience, 14(9), 5147-5159.

Dayan, P. and Abbott, L. F. (2001). Theoretical Neuroscience: Computational and Mathematical Modeling of Neural Systems. MIT Press.

de Garis, H. (1990) Genetic programming: Evolution of time dependent neural network modules which teach a pair of stick legs to walk. In Proc. 9th. Eur. Conf. Artificial Intelligence, 204-206, Stockholm.

DeMarse, T., Wagenaar, D., Blau, A., \& Potter, S. (2001) The Neurally Controlled Animat: Biological Brains Acting with Simulated Bodies. Autonomous Robotics, 11, 305-310. 
DiPaolo, E. (2000) Homeostatic adaptation to inversion of the visual field and other sensorimotor disruptions. In From Animals to Animats 6, SAB 2000, pp.440-449. MIT Press.

DiPaolo, E. (2003) Evolving spike-timing-dependent plasticity for single-trial learning in robots. Phil. Trans. R. Soc. Lond. A, 361:2299-2319.

Doya, K. (2002) Metalearning and neuromodulation. Neural Networks, 15:495-506.

Ekeberg, O. (1993) A combined neuronal and mechanical model of fish swimming. Biological Cybernetics, 69:363-374.

Ekeberg, O., Lansner,A. and Grillner, S. (1995) The neural control of fish swimming studied through numerical simulations. Adaptive Behavior 3(4):363-384.

El-Nashar, H. and Cerdeira, H. (2009) Determination of the critical coupling for oscillators in a ring. Chaos, 19(3), 033127.

Engel, A., Fries, P. and Singer, W.(2001) Dynamic predictions: oscillations and synchrony in top-down processing. Nat Rev Neurosci., 2(10):704-716.

Fernando C, Karishma KK, Szathmáry E (2008) Copying and Evolution of Neuronal Topology. PLoS ONE 3(11): e3775. doi:10.1371/journal.pone.0003775.

Fernando C, Vasas V, Szathmáry E, Husbands P (2011) Evolvable Neuronal Paths: A Novel Basis for Information and Search in the Brain. PLOS ONE 6(8): e23534. doi:10.1371/journal.pone.0023534

Ferree TC, Lockery SR (1999) Computational rules for chemotaxis in the nematode C. elegans. J. Comput Neurosci 6:263-277.

FitzHugh R. (1961) Impulses and physiological states in theoretical models of nerve membrane. Biophysical J. 1:445-466

Floreano, D. and Mondada, F. (1994) Automatic Creation of an Autonomous Agent: Genetic Evolution of a Neural-Network Driven Robot. In D. Cliff, P. Husbands, J. Friston, K. (2000) The Labile Brain. i. Neuronal transients and nonlinear coupling. Phil.Trans. R. Soc. Lond. B, 355:215-236.

Meyer, and S. W. Wilson, (Eds), From Animals to Animats III: Proceedings of the Third International Conference on Simulation of Adaptive Behavior, 402-410. MIT PressBradford Books, Cambridge, MA.

Floreano, D. and Urzelai, J. (2000) Evolutionary Robots with on-line self-organization and behavioral fitness. Neural Networks 13(4-5) 431-443.

Floreano, D. \& Mattiussi, C. (2001) Evolution of Spiking Neural Controllers for Autonomous Vision-Based Robots. In T. Gomi (Ed), Evolutionary Robotic: From Intelligent Robotics to Artificial Life. Tokyo:Springer Verlag. 
Floreano, D., Husbands, P., and Nolfi, S. (2008) Evolutionary robotics. In Siciliano and Khatib (Eds.) Springer Handbook of Robotics, chapter 61, pages 1423-1451, Springer.

Frigg, R. and Hartmann, S. (2008) Models in Science, The Stanford Encyclopedia of Philosophy (Fall 2008 Edition), Edward N. Zalta (ed.), URL =

$<$ http://plato.stanford.edu/archives/fall2008/entries/models-science/>.

Gally, J., Montague, P., Reeke, G., \& Edelman, G. (1990) The NO hypothesis: possible effects of a short-lived, rapidly diffusible signal in the development and function of the nervous system. Proc Natl Acad Sci USA, 87, 3547-3551.

Gerken, W.C., Purvis, L.K. and Butera, R.J. (2005) Use of a Genetic Algorithm for Neuron Model Specification. In Proc. 2nd International IEEE EMBS Conference on Neural Engineering, 304-306, IEEE Press.

Granger CWJ. 1969. Investigating causal relations by econometric models and crossspectral methods. Econometrica 37:424-438.

Grillner, S., Wallen, P. and Brodin,L. (1991) Neuronal network generating locomotor behavior in lamprey: Circuitry, transmitters, membrane properties, and simulation. Annu. Rev. Neurosci. 14:169-199.

Gurkiewicz, M. and Korngreen, A. (2007) A numerical approach to ion channel modelling using whole-cell voltage-clamp recordings and a genetic algorithm. PLoS Comput Biol 3:e169.

Hallam, J. and Ijspeert, A. (2003) Using Evolutionary Methods to Parameterize Neural Models: a Study of the Lamprey Central Pattern Generator. In R. J. Duro, J. Santos and M. Grana (Eds.), Biologically inspired robot behavior engineering, Springer, p. 119142.

Hartmann, S. (1995), Models as a Tool for Theory Construction: Some Strategies of Preliminary Physics. In W. Herfel et al. (Eds) Theories and Models in Scientific Process. (Poznan Studies in the Philosophy of Science and the Humanities 44.) Amsterdam: Rodopi., 49-67.

Harvey, I., Husbands, P. and Cliff, D.(1993) Issues in evolutionary robotics. In From Animals to Animats 2: Proc. 2nd Int. Conf. on the Simulation of Adaptive Behaviour, Meyer, J., Roitblat, H. and Wilson, S. (Eds.), pp.364-373, MIT Press.

Harvey, I., Husbands, P., and Cliff, D. (1994) Seeing the light: Artificial evolution, real vision. In D.T. Cliff, P. Husbands, J.-A. Meyer, and S. Wilson (Eds), From Animals to Animats 3: Proceedings of the Third International Conference on Simulation of Adaptive Behaviour, SAB94, 392-401. MIT Press, Cambridge, Massachusetts.

Harvey, I., Di Paolo, E., Wood, R., Quinn, M. and Tuci, E. (2005). Evolutionary robotics: a new scientific tool for studying cognition. Artificial Life, 11(1-2):79-98. 
Held, R. and Hein, A. (1963) Movement-produced stimulation in the development of visually guided behavior. Journal of Comparative and PhysiologicalPsychology, 56(5):872-876.

Hodgkin, A., and Huxley, A. (1952): A quantitative description of membrane current and its application to conduction and excitation in nerve. J. Physiol. 117:500-544.

Husbands, P. (1992) An Ecosystems Model for Integrated Production Planning. Int. Journal of Computer Integrated Manufacturing 6(1\&2), 74--86.

Husbands, P. and Harvey, I. (1992) Evolution versus design: controlling autonomous mobile robots. Proc. 3rd Annual Conf. on Artificial Intelligence, Simulation and Planning in High Autonomy Systems. IEEE Computer Society Press, Los Alimitos, CA, 139-146.

Husbands, P., Harvey, I., Cliff, D. and Miller, G. (1997) Artificial Evolution: A new path for AI? Brain and Cognition 34, 130-159.

Husbands, P. (1998) Evolving robot behaviours with diffusing gas networks. In: Husbands, P. and Meyer, J.-A. (Eds). Evolutionary Robotics: First European Workshop, EvoRobot98, Lecture Notes in Computer Science 1468; 71-86, Springer.

Husbands, P., Smith, T., Jakobi, N., \& O'Shea, M. (1998) Better Living through Chemistry: Evolving GasNets for Robot Control. Connection Science, 10(3\&4), 185210.

Husbands, P. and Philippides, A. and Smith, T. and O'Shea, M. (2001) The shifting network: Volume Signalling in Real and Robot Nervous Systems In Kelemen, J. and Sosik, P. (Eds.) Proc. ECAL2001, LNAI 2159, Springer, 23-37.

Husbands, P., Philippides, A., Vargas,P., Buckley,C., Fine,P., Di Paolo, E., \& O'Shea, M. (2010) Spatial, temporal and modulatory factors affecting GasNet evolvability in a visually guided robotics task, Complexity 16(2):35-44.

Ijspeert, A., Hallam, J. and Willshaw, D. (1999) Evolving swimming controllers for a simulated lamprey with inspiration from neurobiology. Adaptive Behavior 7(2):151-172.

Ijspeert, A., Crespi, A., Ryczko, D. and Cabelguen, J. (2005) From swimming to walking with a salamander robot driven by a spinal cord model. Science, 315(5817):1416-1420.

Izhikevich, E. (1999) Weakly pulse-coupled oscillators, FM interactions, synchronization, and oscillatory associative memory. IEEE Trans. Neural Networks, 10(3):508-526.

Izhikevich, E. (2007) Dynamical Systems in Neuroscience: The Geometry of Excitability and Bursting, MIT Press. 
Izquierdo, E. (2006) The dynamics of learning behaviour: a situated, embodied, and dynamical systems approach. $\mathrm{PhD}$ thesis, Centre for Computational Neuroscience and Robotics, University of Sussex.

Izquierdo, E.J. and Lockery S.R. (2010) Evolution and analysis of minimal neural circuits for klinotaxis in Caenorhabditis elegans. Journal of Neuroscience 30:1290812917.

Jakobi, N. (1998) Running across the reality gap: Octopod locomotion evolved in a minimal simulation. In P. Husbands \& J.-A. Meyer, (Eds), Evolutionary Robotics: First European Workshop, EvoRobot98 (pp. 39-58). Berlin: Springer.

Katz, P. (Ed.) (1999) Beyond Neurotransmission: Neuromodulation and its Importance for Information Processing. Oxford: Oxford University Press.

Kelso, J.A.S. (1995). Dynamic patterns: The self-organization of brain and behavior. MIT Press.

Kodjabachian, J. and Meyer, J,-A. (1998) Evolution and development of neural networks controlling locomotion, gradient following and obstacle avoidance in artificial insects. IEEE Trans. Neural Networks,9:796-812.

Konig, P., Engel, A. and Singer, W. (1996) Integrator or coincidence detector? The role of the cortical neuron revisited. Trends in Neurosciences, 19:130-137.

Korn, H. \& Faure, P. (2003). Is there chaos in the brain? II. Experimental evidence and related models. Comptes Rendus Biologies, 326, 787-840.

Kuniyoshi, Y. \& Suzuki, S. (2004). Dynamic emergence and adaptation of behavior through embodiment as coupled chaotic field. In Proceedings of IEEE International Conference on Intelligent Robots and Systems, pages 2042-2049.

Kuniyoshi Y, Sangawa S (2006) Early Motor Development from Partially Ordered Neural-body Dynamics: Experiments with a Cortico-spinal-musculo-skeletal Model. Biol Cybern 95:589-605.

Kuramoto, Y. (1984) Chemical Oscillation, Waves, and Turbulence. Springer, New York.

Li, Z. and Hopfield, J. (1989) Modeling the olfactory bulb and its neural oscillatory processings. Biological Cybernetics, 61(5):379-392.

McHale, G. and Husbands, P. (2004) Gasnets and other evovalble neural networks applied to bipedal locomotion. In Schaal, S. et al. (Eds) Proc. From Animals to Animats 8: Proceedings of the Eigth International Conference on Simulation of Adaptive Behavior (SAB'2004), 163-172, MIT Press. 
Moioli, R., Vargas, P., and Husbands, P. (2010) Exploring the Kuramoto model of coupled oscillators in minimally cognitive evolutionary robotics tasks. In Proc. IEEE Congress on Evol. Comput 2010 (CEC'10), IEEE Press. DOI:

10.1109/CEC.2010.5586486

Moioli, R., Vargas, P., and Husbands, P. (2012) Synchronisation effects on behavioural performance and information dynamics of a simulated robotic agent, Biological Cybernetics 106(6-7):407-427.

Monteiro, L. Canto,N., Orsatti,J . and Piqueira, F.. (2003) Global and partial synchronism in phase-locked loop networks. IEEE Transactions on Neural Networks, 14(6).

Mpitsos, G.J., Burton, R.M., Creech, H.C., \& Soinila, S.O. (1988). Evidence for chaos in spike trains of neurons that generate rhythmic motor patterns. Brain Research Bulletin, 21, 529-538.

Smith, T., Husbands, P., Philippides, A., and O’Shea, M. (2002) Neuronal plasticity and temporal adaptivity: Gasnet robot control networks. Adapt Behav. 10:161-184.

Nolfi, S. and Floreano, D. (2000) Evolutionary Robotics: The Biology, Intelligence, and Technology of Self-Organizing Machines. Cambridge, MA: MIT Press/Bradford Books.

North, G. and Greenspan, R. (Eds.) (2007) Invertebrate Neurobiology, Cold Spring Harbor Monograph Series 49, Cold Spring Harbor Laboratory Press.

Parisi D. \& Nolfi S. (1993). Neural network learning in an ecological and evolutionary context. In V.Roberto (Ed.), Intelligent perceptual systems. Berlin: Springer, pp. 20-40.

Pfeifer R and Bongard J (2007) How the Body Shapes the Way We Think: A New View of Intelligence, MIT Press/Bradford Books.

Philippides, A., Husbands, P., \& O'Shea, M. (2000) Four Dimensional Neuronal Signaling by Nitric Oxide: A Computational Analysis. Journal of Neuroscience, 20(3), 1199-1207.

Philippides, A., Husbands, P., Smith, T., \& O’Shea, M. (2003) Structure based models of NO diffusion in the nervous system. In J.Feng(Ed.), Computational Neuroscience: $a$ comprehensive approach (pp. 97-130). London: Chapman and Hall/CRC Press.

Philippides, A., Ott, S., Husbands, P., Lovick, T., \& O’Shea,M. (2005a) Modeling cooperative volume signaling in a plexus of nitric oxide synthase-expressing neurons. Journal of Neuroscience, 25(28), 6520-6532.

Philippides, A., Husbands, P., Smith, T., \& O’Shea, M. (2005b) Flexible couplings: diffusing neuromodulators and adaptive robotics. Artificial Life, 11(1\&2), 139-160. 
Pinaud, R., Tremere, L. and De Weerd, P. (Eds) (2006). Plasticity in the visual system: from genes to circuits. New York: Springer.

Pitti A, Niiyama R, Kuniyoshi Y (2010) Creating and Modulating Rhythms by Controlling the Physics of the Body. Auton Robot 28(3):317-329.

Pollack., J. (2012) Mindless Intelligence. In Vargas et al. (Eds) The Horizons of Evolutionary Robotics, MIT Press (this volume).

Reil, T., \& Husbands, P. (2002) Evolution of central pattern generators for bipedal walking in real-time physics environments. IEEE Trans. Evolutionary Computation, 6(2), 10-21.

Santos, B., Barandiaran, X. and Husbands, P. (2012) Synchrony and Phase Relation Dynamics Underlying Behaviour, Adaptive Behavior 20(5):321-336.

Seth, A. K., McKinstry, J.L., Edelman, G.M. and Krichmar, J.L. (2004) Visual binding through reentrant connectivity and dynamic synchronization in a brain-based device. Cerebral Cortex, 14(11): 1185-1199.

Seth, A. (2005) Causal connectivity of evolved neural networks during Behaviour, Network: Computation in Neural Systems, 16(1): 35-54.

Seth, A.K., Barrett, A.B., and Barnett. L. (2011). Causal density and integrated information as measures of conscious level. Phil Trans R. Soc. A. 369:3748-3767

Shim, Y. and P. Husbands (2010) Chaotic Search of Emergent Locomotion Patterns for a Bodily Coupled Robotic System. In Proc. Alife XII, MIT Press, 757-764.

Shim, Y. and Husbands, P. (2012) Chaotic Exploration and Learning of Locomotion Behaviours. Neural Computation 24(8):2185-2222.

Singer, W. (1999) Neuronal synchrony: a versatile code for the definition of relations? Neuron, 24(1):49-65.

Schmidhuber, J., Wierstra, D., Galiolo, M. and Gomez, F. (2007) Training recurrent networks by Evolino. Neural Comput., 19(3):757-779.

Smith, T.M.C., Husbands, P., Philippides, A., \& O'Shea, M. (2002) Neuronal plasticity and temporal adaptivity: GasNet robot control networks. Adaptive Behavior, 10(3/4), 161-184.

Smith, T.M.C., Husbands, P., \& O'Shea, M. (2003) Local evolvability of statistically neutral GasNet robot controllers. Biosystems, 69, 223-243.

Smith, M. (Ed) (2007) Stelarc:The Monograph, MIT Press.

Steingrube, S., Timme, M., Worgotter, F., \& Manoonpong, P. (2010) Self-organized adaptation of a simple neural circuit enables complex robot behaviour. Nature 
Physics, 6, 224-230.

Suzuki, M., Floreano, D. and DiPaolo, A. (2005) The Contribution of Active Body Movement to Visual Development in Evolutionary Robots. Neural Networks, 18(5/6):656-665.

Tononi, G., Sporns, O. and Edelman, G. (1992) Reentry and the problem of integrating multiple cortical areas: Simulation of dynamic integration in the visual system. Cerebral Cortex, 2:310-335.

Tononi, G., Sporns, O., \& Edelman, G. (1999) Measures of degeneracy and redundancy in biological networks. Proc. Natl. Acad. Sci. USA, 96, 3257.

Varela, F., Thompson, E. and Rosch, E. (1991) The Embodied Mind, MIT Press.

Varela, F., Lachaux, J., Rodriguez, E. and Martinerie, J. (2001) The brainweb: phase synchronization and large-scale integration. Nat. Rev. Neurosci., 2(4).

Vargas, P., Moioli, R., Castro, L., Timmis, J., Neal, M. and Von Zuben, F. (2005) Artificial homeostatic system: a novel approach. In Proceedings of the VIIIth European Conference on Artificial Life, Springer.

Vargas, P., Moioli, R., Von Zuben, F., \& Husbands, P. (2009) Homeostasis and Evolution Together Dealing with Novelties and Managing Disruptions. International Journal of Intelligent Computing and Cybernetics, 2(3), 435-454.

Vaughan, E., Di Paolo, E., \& Harvey, I. (2004) The evolution of control and adaptation in a 3D powered passive dynamic walker. In J. Pollack, M. Bedau, P. Husbands, T. Ikegami, \& R. Watson, (Eds), Proceedings of the Ninth International Conference on the Simulation and Synthesis of Living Systems, Artificial Life IX (pp. 139-145). Cambridge, MA: MIT Press.

Von der Malsburg, C. (1981) The correlation theory of the brain. Internal report. MaxPlanck-Institute for Biophysical Chemistry, Gottingen, Germany.

Webb, B. (2001). Can robots make good models of biological behaviour? Behavioural and Brain Sciences, 24 (6), 1033-1050.

Webb, B. (2009) Animals versus animats: or why not model the real iguana? Adaptive Behavior , 17(4), 269-286.

Wheeler, M. (2005) Reconstructing the Cognitive World. Cambridge, MA: MIT Press. Wiley, D., Strogatz, S. and Girvan, M. (2006) The size of the sync basin. Chaos, 16, 015103.

Wimsatt, W. (2002) Using False Models to Elaborate Constraints on Processes: Blending Inheritance in Organic and Cultural Evolution. Philosophy of Science, 69(3), Supplement: Proceedings of the 2000 Biennial Meeting of the Philosophy of Science Association. Part II: Symposia Papers, pp. S12 -S24. 
Wood, J., \& Garthwaite, J. (1994) Model of the diffusional spread of nitric oxide implications for neural nitric oxide signaling and its pharmacological properties. Neuropharmacology, 33, 1235-1244. 